\title{
Network Analysis of Internal Migration in Austria
}

\author{
DINO PITOSKI, THOMAS J. LAMPOLTSHAMMER, and PETER PARYCEK, Danube University \\ Krems
}

\begin{abstract}
Human migration, and urbanization as its direct consequence, are among the crucial topics in regional and national governance. People's migration and mobility flows make a network structure, with large cities acting as hubs and smaller settlements as spokes. The essential method by which these phenomena can be analyzed comprehensively is network analysis. With this study, we first contribute to capacity building regarding the analysis of internal (national) migration data by providing a set of network indicators, models, and visualizations tested and argued for in terms of applicability and interpretability for analyzing migration. Second, we contribute to the understanding of the shape and scale of the phenomenon of internal migration, particularly toward urbanization and mobility flows between human settlements (i.e., cities, towns, and villages). Third, we demonstrate the utility of our approach on the example of internal migration flows in Austria on the settlement level and provide a longitudinal analysis for the period from 2002 to 2018. To the best of our knowledge, this is the first time that the key traits of a network of internal migration are identified for a European country, which, when accompanied by additional country analyses, has the potential to reveal the migration patterns in the region and beyond.
\end{abstract}

CCS Concepts: • Applied computing $\rightarrow$ E-government; • Theory of computation $\rightarrow$ Social networks;

Additional Key Words and Phrases: Network science, internal migration, sustainability, policymaking

ACM Reference format:

Dino Pitoski, Thomas J. Lampoltshammer, and Peter Parycek. 2021. Network Analysis of Internal Migration in Austria. Digit. Gov.: Res. Pract. 2, 3, Article 25 (July 2021), 24 pages.

https://doi.org/10.1145/3447539

\section{INTRODUCTION}

The latest mass migration to Europe, often cited as the "migration crisis," showed in an unambiguous way the exigence for international joint policy actions across all geographic scales [1]. This circumstance is also reflected in the fact that the 2030 Agenda for Sustainable Development, comprising the 17 Sustainable Development Goals (SDGs), explicitly includes the challenges of migration $[2,3]$ that become directly embedded into regional and national political strategies.

DP and TJL conceptualised the paper and the methodology, DP carried out the analyses, DP and TJL drafted the initial manuscript. DP, TJL, PP revised the draft, PP was responsible for senior supervision and funding acquisition. All authors have read and agreed to the published version of the manuscript. Regarding the sequence of authors, the "sequence-determines-credit" (SDC) approach was applied.

The authors declare no conflict of interest.

This work was co-financed by the Asylum, Migration, and Integration Fund and the Austrian Federal Ministry of the Interior (grant BMIIA123004/0046-III/5/a/2016).

Author's address: D. Pitoski (corresponding author), T. J. Lampoltshammer, and P. Parycek, Department for E-Governance and Administration, Danube University Krems, Dr.-Karl-Dorrek-Straße 30, 3500, Krems an der Donau, Austria; emails: dino.pitoski@donau-uni.ac.at, thomas.lampoltshammer@donau-uni.ac.at, peter.parycek@donau-uni.ac.at. 
The targets (indicators) defined by the SDGs are heavily reliant on data collection, particularly by national authorities [4, pp. 8-9]. These data are often translated one-to-one as indicators, and indicators are often rated as reductionist [5]. It becomes clear to the development community that more efforts need to be invested into building national capacities to systematically analyze migration data [6].

With current capacities, aided by politicization and media influence, the scale of migration is oftentimes been wrongly perceived (i.e., to a larger extent than there actually is) [6]. Often, and particularly in global development policy, the international dimension of migration gets the most attention. Migration, in the minds of many, reflects relocation to a different country. The fact is, however, that the share of international migrants in the global population, persistently, from the start of its measuring (1960s onward), stays at about 3\% [7], whereas within-country migration-internal migration-is known to be at least three times greater than international [8]. Although the two types of migration are proven to be complementary, where one is a strong predictor of the other [9], the research on internal migration remains largely ignored [10].

Moreover, migration and urbanization are often considered as separate topics. The rate of urbanization is enormous, with the number of people living in cities projected to rise to about 5 billion people by 2030-yet another global problem requiring collaborative governance (SDG 11: Sustainable cites and communities). Urbanization is a direct result of migration, be it internal or international, and one could argue that urbanization is just another class from the utterly many categories of migration [11]. Policy experts, however, become more and more aware that internal and international migration, mobility, and urbanization must be approached integratively [12]. In the European Union, especially, due to its constitution being founded on the free movement of people, migration and mobility have for a long time been recognized as a unitary concept [13]. Increasingly, EU policies also recognize the connection of migration (mobility) and urbanization, with migration being one of the cross-cutting issues consequential for urban development [14].

What underlies all migration, international and internal, is a network of cities, towns, and villages (human settlements), in which large cities act as hubs and smaller settlements as spokes. By analyzing this network one can obtain relevant insights about urbanization as well, as smaller settlements become larger settlements and vice-versa inherently as a consequence of internal migration, alongside international migration and natural birth. In addition, analyzing migrations within shorter time spans practically means analyzing mobility. In that perspective, to support the policies in any of the relative domains-governance of migration and mobility, or urban growth planning-both national and international capacities need to be improved to incorporate comprehensive analyses of network patterns, and forecasting of migration exchange in the network of human settlements. Effectively, this is pursuable using tools of network science.

Network analyses of human migration, especially at the level of human settlements, are, however, very rare. From the beginning of the 2010s, with the opening of the global migration database [15], several network analyses have been done at the level of countries, describing global connectedness of countries in terms of bilateral migrant stocks. These analyses are, however, overshadowed by uncertainties in terms of the accuracy of data, which are clearly addressed in the original source, where data can be considered to provide only a rough estimate of global nation-in-nation dispersion. Consequently, the network analyses based on these data are neither reliable as policy information nor suitable for focused policy decisions. However, only a few network studies have looked into migration at the level of human settlements, with the more accurate and, also important, more fine-grained and regularly reported data on migration flows. The majority of these studies analyze internal migration in the United States, followed by migration between Chinese larger cities, although none have yet investigated migration within any of the European countries. At the same time, both international and internal migration analyses have been run as trial applications of a complex network approach to migration and are laden with methodological uncertainty [16].

Europe, particularly the European Union, might be a very suitable ground for a novel, refined network analysis of inter-settlement migration, whereby successively explaining internal migration, country by country, we might understand migration within the region as a whole. This would be due to the adoption of common 
policies that regulate migration, mobility, urbanization, and all other domains of living, and which presumably all lead toward the convergence of relocation patterns within and between societies. Moreover, the data collection and warehousing policies strive toward unification across the region [17], so conjoining the various datasets, and subsequently, analyses, would be relatively easily achieved. Data on internal migrations are collected by membership states in a way that they are reliable, timely, and fine grained, showing exact relocation counts at the level of human settlements-cities, towns, and villages. Such data are, for instance, immediately available for the Republic of Austria from Statistics Austria [18].

In terms of all of the issues exposed, there are three main goals aimed for by the study at hand:

(1) To contribute to capacity building regarding the analysis of internal (national) migration data. We do this by providing a set of indicators, models, and visualizations, which we tested and argued for in terms of applicability and interpretability in the case of a network analysis of migration, emphasizing the crucial aspect of interpretability of the selected indicators for policymakers.

(2) To contribute to the understanding of the shape and scale of the phenomenon of internal migration, particularly toward urbanization and mobility flows between human settlements (i.e., cities, towns, and villages).

(3) To demonstrate our approach on the example of internal migration flows in Austria on the settlement level, and to provide a longitudinal analysis for the period from 2002 to 2018 .

We advise that a thorough, settlement-level analyses like ours could serve as pieces of puzzle that build the view into the interconnectedness of cities, and human settlements in general, in Europe, as well as globally. To the best of our knowledge, this is the first attempt to run a network analysis of migration in a European country at such a detailed level, both in terms of data and in terms of network methodology. We consider our analysis as contributing to the national and global sustainable development policy in the perspective, not only in the part of migration and mobility but also in the part of urbanization and urban growth planning. At the least, our analysis provides Austrian national governors and policy experts with an insight into the structure and development of country's internal migration.

The rest of this article is structured as follows. In Section 2, we provide a review of previous network analyses on human migration in the context of network-scientific research in general. In Section 3, we define the network of internal migration in Austria, its underpinning data, and the summary and schedule of the methodology deployed in the analysis. In Section 4, we analyze the network and discuss the methodological issues, along with the results. In Section 5, we recapitulate the main findings from the applications. We conclude with Section 6, where we summarized the motivations and contributions, and point to the avenues open(ed) for further research.

\section{RELATED WORKS}

The field of network science has seen an exponential growth of scientific contributions [19] since emergence of some seminal works in the late 1990s [20,21]. The migration phenomenon, however, received greater attention from network scientists only very recently. In the last 3 years, more than a dozen new applications have emerged compared to the handful of studies from the beginning of decade. All of these studies essentially were the test runs of a network approach to migration. They were based on hands-on implementations of different network measures and models, and were not touching upon the methodological issues prior to their application-issues such as the appropriate network abstractions from migration data, applicability of different network measures and models to the selected network abstractions, or the interpretability of the selected network tools for eventual use in practice.

The methodology of network science is generally not of a fixed design. There is no comprehensive theoretical guide that sorts and recommends particular measures and models for their application on particular network abstractions. Base network-theoretical research is largely concentrated on artificial networks, which are almost exclusively binary network abstractions, in which only the existence of flow is accounted for, and not its actual size; if any flow between two nodes exists, a link between these nodes exists, and its weight essentially is 
1 (person). All prevalent network indicators have essentially originated from these binary network abstractions (see the work of Newman et al. [22] for an overview of the field). Weighted observations, in which the actual size of inter-nodal flow is accounted for, are still largely under-represented in both theoretical and applied network research, and the selection of indicators and models to suit the weighed abstractions is often dependent upon specific application. Human migration is one study case where weighted network abstraction is required when one desires realistic and practical results from the analysis. In this observation, locations are nodes connected by directed links (weights) representing batches of people moving between locations. In former network studies on migration, there was usually no differentiation between binary and weighted network abstractions, or between indicator and models as being applicable for one abstraction or the other. We cite and provide some very general findings of these past studies in the sequel. The systematic process by which this literature was gathered, including a detailed analysis of the formerly applied network methods, is described in detail in our related work [16].

Among the first studies that deployed a network analysis to the phenomenon of migration were those of Fagiolo and Mastrorillo [23], Davis et al. [24], and Tranos et al. [25], basing the global migration network on the data estimated by the United Nations' Department of Economic and Social Affairs on international bilateral migrant stocks [26]. The studies found a steady enhancement in the small-world effect, high network clustering, and a robust community structure throughout the period from 1960 to 2000. Stable interconnectedness was found at the top of the network hierarchy also by Peres et al. [27], with developed countries of the West acting as the great attractors of migration from an increasingly diverse array of origin countries. Similar findings were brought forward by Porat and Penguigui [28], who talked about the division into two separated groups of countries with large (about 20\%) and small (about $80 \%$ ) degree of connectedness. A more recent study by Danchev and Porter [29] took a closer look into community structures and found a heterogeneous structure in the year 2000, where some communities are increasingly globally integrated and some geographic regions largely isolated. Other studies [30, 31] investigated more recent periods (2013 and 2015, respectively) and refined the differences in influence of particular countries expanding the centrality indicators to incorporate the economic component into connectivity. A more comprehensive investigation including factors other than economic through combining network and statistical approaches was performed by Windzio [32] for the period from 1990 to 2013. All studies noted a hierarchy of attractiveness between countries, with the direction of migration running from poor to rich countries.

An inter-country analysis was also conducted for migration between European countries [33], again deploying data on bilateral migrant stocks. The studies observed the periods from 1974 to 2004 and 2005 to 2013, and reported a generally increased interconnectedness among countries, but with a much more pronounced richclub pattern for the European network with respect to the worldwide network.

Few studies have concentrated on U.S. inter-county migration using Internal Revenue Service data and changes of address on tax returns. The study by $\mathrm{Xu}$ [34] reported on fundamental hub-and-spoke disassortative structure of the U.S. network (1990-2011), with a small number of highly connected metropolitan hubs exchanging high-volume and long-distance migration, and less connected areas attaching to these with smaller migration volumes. In the work of Liu et al. [35], the same was noted for the periods from 1992 to 1993 and 2012 to 2013, with a steady diversity of destinations but a steady increase in the average size of migration between best-friend connections. Most recently, U.S. inter-county migration in the period from 2000 to 2015 was analyzed by Goldade et al. [36] and Charyyev and Gunes [37]. The authors reported a significant majority of migration being within U.S. states and a greater variation in destination counties in inter-state migration.

A network analysis by $\mathrm{Li}$ et al. [38] was dedicated to explaining migration between urban agglomerations in the Chinese mainland in 2000 and 2010. Small-world effect and clustering are reported to have aggravated for urban agglomerations, with short migration distances dominating the links. In the work of Sun and Pan [39] the network of inter-city migration for Chinese graduates in 2011 was analyzed using census data, with the 
emphasis to economic attributes of each city, reporting again a clear power law scaling and a tendency of this migrant group to move toward urban province capitals.

A few additional works published mainly as working papers, such as those that investigate migration within the United States [40-42], migration within the United Kingdom [43], and internal migration in Mexico [44], were found while systematically gathering the literature in our earlier work [16]. Few additional works also emerged as closely related due to their employment of some of the network science tools, although not primarily observing migration. These include works that investigate the relation of computer technologies including social media on human mobility [45-47], the relation of migration and the spread of diseases [48, 49], the relation of migration and income/trade/remittances [50-53], and those that compare migration and tourism flows [54, 55]. To avoid making this review too long, we leave it to the reader to consult these sources for their general findings.

In terms of the spatial scope of the previous studies, the literature review clearly informs about a shortage of the analyses on internal migration, at the level of settlements, and especially on any European country. In terms of the network methodology, in this same review we identified and ranked by frequency of application all of the network measures and models utilized in former studies. In this way, we produced a set of measures that are straightforwardly applicable and interpretable after application to migration, as well as those whose applicability and interpretability has to yet be established. Essentially, what we derived from the review is the network methodology appropriate for the analysis of migration, since, as explained at the beginning of this section, a common methodology for the exploration of networks does not exist.

A comprehensive overview of network measures with the frequency of their application to the phenomenon of migration, as well as the overview of the network abstractions on which these measures were applied, is provided in our earlier work [16]. There, a thorough theoretical argumentation is also made on each measures' applicability in terms of the appropriate network abstractions, their interpretability, and their potential usability in the domain of policymaking. In the subsequent analysis, we apply directly the set of measures outlined in that review, simultaneously discussing other important topics raised in the theoretical argumentation, such as network abstraction, reduction of scope and generalization, and the information delivered by each measure, now after being applied to our concrete case. In the next section, we define in mathematical terms the analyzed network, as well as the data from which it has been abstracted, and unfold the precise methodology (i.e., network indicators and models) that we employ for this analysis, and which stems from the literature review presented previously.

\section{DATA AND METHODOLOGY}

Statistics Austria defines internal migration in any given year as the count of official changes of address of residence occurring on each link between/within municipalities in that same year. These address changes, independent of the length of stay at any given address, count as migration if there is a minimum stay of 90 days in the country as a whole. The original data used in the analysis are made publicly available [18], and the detailed outline of definitions, methods of data collection, and quality of data is provided at Bundesanstalt Statistik Österreich [56]. Based on these data and definitions, here we define the Austrian internal migration network as a weighted directed graph $\mathcal{G}_{t}=\left(\mathcal{N}, \mathcal{L}_{t}, \mathcal{W}_{t}\right)$, whose

- nodes $\mathcal{N}=\left\{n_{1}, n_{2}, \ldots, n_{N}\right\}$ represent all Austrian municipalities,

- link weights $\mathcal{W}_{t}=\left\{w_{i j}\right\}_{N \times N}, i, j=1, \ldots, N$, where $i$ can be equal to $j$, are the counts of official changes of address of residence from municipality $i$ to municipality $j$ in year $t, t=2002, \ldots, 2018$, and

- links $\mathcal{L}_{t}=\left\{l_{i j_{t}}\right\}_{N \times N}$ is a binary projection of $\mathcal{W}_{t}$ such that $l_{i j_{t}}=1$ if $w_{i j_{t}}>0$ and $l_{i j_{t}}=0$ if $w_{i j_{t}}=0$.

In the most general formulation, we take into account loops $\left(w_{i i t} \geq 0\right)$ as they occur in the real world (intramunicipal migrations). From $\mathcal{G}_{t}$, we can further identify a subgraph $\mathcal{G}^{\prime}{ }_{t}=\left(\mathcal{N}, \mathcal{L}^{\prime}{ }_{t}, \mathcal{W}^{\prime}{ }_{t}\right)$, where $\mathcal{W}^{\prime}{ }_{t}=\mathcal{W}_{t} \backslash$ $\left\{w_{i i t}\right\}$ and $\mathcal{L}^{\prime}{ }_{t}$ is the according binary projection of $\mathcal{W}^{\prime}{ }_{t}$, and which essentially is a subnetwork of $\mathcal{G}_{t}$ based on exclusively inter-municipal migrations. 
The preceding definitions refer to total internal migrations per year. The original dataset, however, contains yearly migration counts reported separately for nationals versus non-nationals, and males versus females, which allows for separate analyses and comparisons of these four group networks, both in $\mathcal{G}_{t}$ and $\mathcal{G}_{t}^{\prime}$. Respective changes in formal notations that discern on the exact levels analyzed or discussed will be denoted in index form such that " $\mathrm{A}$ " will designate Austrian nationals, "O" will designate non-nationals, "M" will designate males, and "F" will designate females.

There is also variability in terms of the granularity at which data are being maintained by Statistics Austria. Namely, the original dataset shows migrations at the municipal level, except for Vienna, where migration is counted at the level of its 23 districts. As migration at the district level has not been reported for other larger municipalities (e.g., Graz and Klagenfurt am Wörthersee, which also comprise multiple municipal districts), we flattened these levels by contracting Vienna districts to a single node (Wien).

All abstracted networks subsequently are composed of 2096 nodes (municipalities). The summary statistics on the weights and links are provided in the supplementary material [60], which also contains the calculated values for the indicators and models that we employ in the following analysis. A summary and schedule of the methodology-indicators and models that we employ in the analysis-is as follows:

(1) Overall descriptives of the main components of migration (inter-municipal and intra-municipal migration), and the description of their inherent relation with population. Here, we assess the sizes and temporal progression of the three categories as a whole and at the level of each municipality. We employ network graphs to grasp the the evolution of the network and correlation analysis for reduction of the temporal scope.

(2) Application of node centrality indicators, namely node strength and degree centrality, and PageRank and HITS algorithm centrality, by which applications we assess attractive or repulsive potential of each municipality in the network. By the analysis of correlations between these indicators, we further evaluate if reduction of the spatial scope of the analysis is possible.

(3) Application of network structural metrics, namely reciprocity, assortativity, transitivity, and community detection algorithms, to describe the general network structure and identify constitutive communities.

(4) Application of network models in terms of link weight estimation, namely the Gravity Law model and the Radiation model, to test their predictive power in the case of internal migration forecasting for Austria.

All of the metrics that constitute our methodology are derived from a systematic literature review made in our earlier work [16], as explained in the previous section. For each indicator or model employed in the sequel, we refer to the original network scientific study from which it has emerged, also using the study's original notation. For more description on each of these network tools and their general interpretation when applied to migration, consult our earlier work [16].

\section{ANALYSIS}

\subsection{Austrian Internal Migration 2002-2018: General Characteristics and the Reduction of Temporal Scope}

Before we start to analyze our network using network metrics, we first analyze and inform on some general characteristics of Austrian internal migration, including its evolution and its innate relationship with population, all with the aim of reducing the temporal scope of the analysis. In the process, we also pull some parallels with migration theory.

The size of migration in a particular country or a region is commonly approximated as $\frac{\sum_{i} w_{i j_{t}}}{\sum_{i} p_{i_{t}}}$, where $p_{i_{t}}$ is a municipality's population at a year's end. For Austria, this share has increased steadily from about $8 \%$ in 2002 to about $9 \%$ in $2018\left(\sum_{i} w_{i j_{2002}}=649153, \sum_{i} w_{i j_{2018}}=797666\right)$. This share of migration in population is rather high when compared to about $2.75 \%$ to $3.25 \%$ as reportedly the size of global international migration in the global 

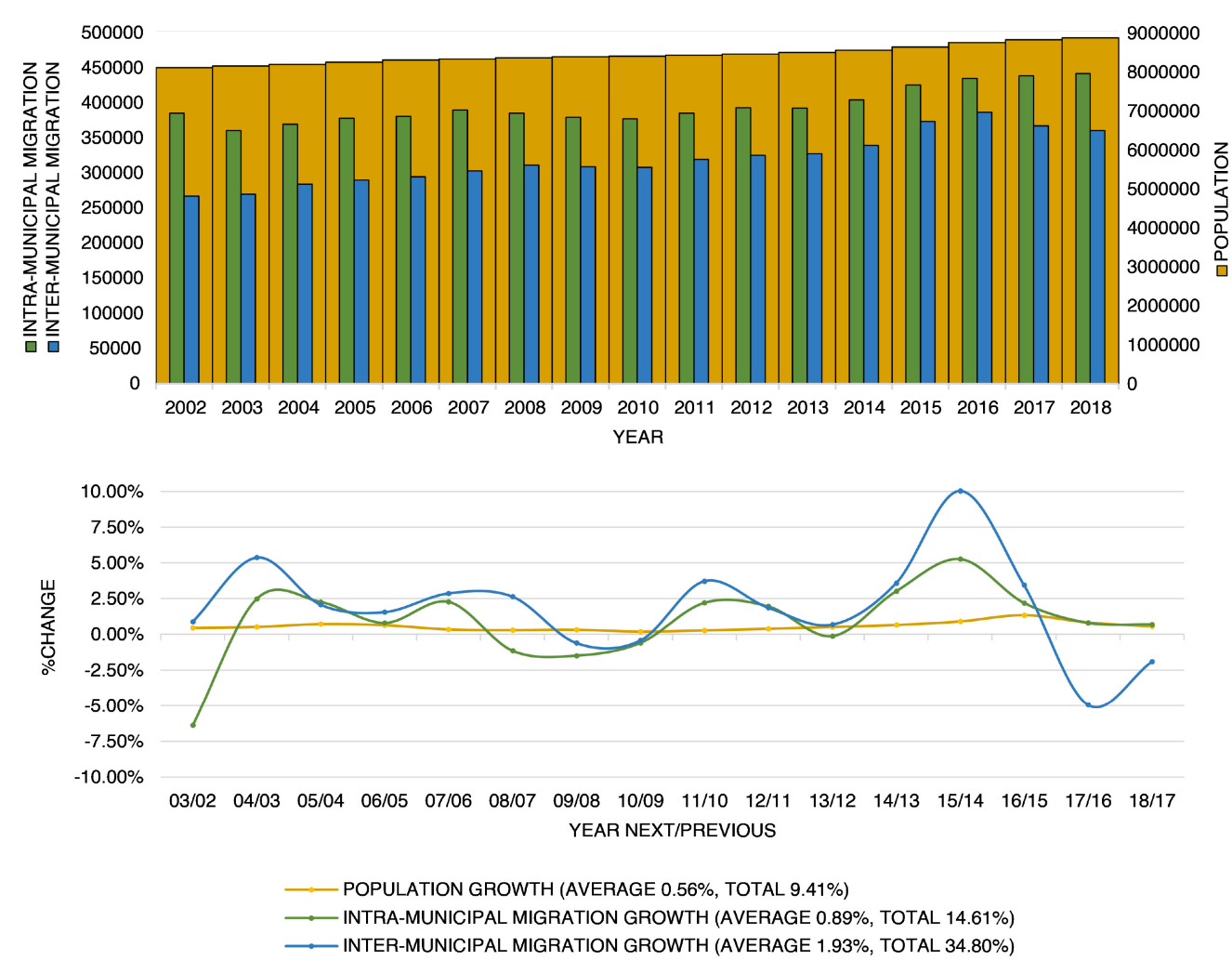

Fig. 1. Sizes and growth rates of population, intra-municipal migration, and inter-municipal migration in Austria from 2002 to 2018. Top: Number of persons counted as migrating/residing within Austria by the end of the specified period. Bottom: Percentage rise or fall in the number of persons migrating/residing in the country with regard to the prior period.

population (see De Haas [7]), but at the same time rather low when compared to the level of global internal migration in the global population of about $11.75 \%$. The latter is evaluated using the last known "conservative" estimate for 2009 from UNDP [8] and the global population estimate for the same year published by the World Bank [57].

In any year of observation, more than half of relocations in Austria (55\% on average) are relocations within one and the same municipality, or intra-municipal migrations. The rest are migrations between different settlements, or inter-municipal migrations. This matches to some extent the fact that there is globally more internal migration activity than international migration activity-about 214 million international migrants compared to about 740 million internal migrants in 2009 (sources same as earlier)-that is, if we analogize global international migration with Austrian inter-municipal migration, and global internal migration with Austrian intra-municipal migration.

The relationships of Austrian inter-municipal migration, intra-muncipal migration, and population, in terms of the overall size and growth of these three categories over years, are provided in Figure 1. One can observe a higher growth of inter-municipal migration relative to intra-municipal migration and, overall, a steady rise of the migration phenomenon.

The relative proportions of these three categories are consistent at the level of individual municipalities. In Figure 2, we provide the distributions of intra-municipal migration, inter-municipal migration, and municipal population, overlapped for each year of the investigated period and ranked according to the average inter-municipal migration over the period. The figure captures the very strong correlations existing among values and value rankings of these three categories, both within and across different yearly periods ( $\min \rho \approx 0.94$ ). Inter-municipal 


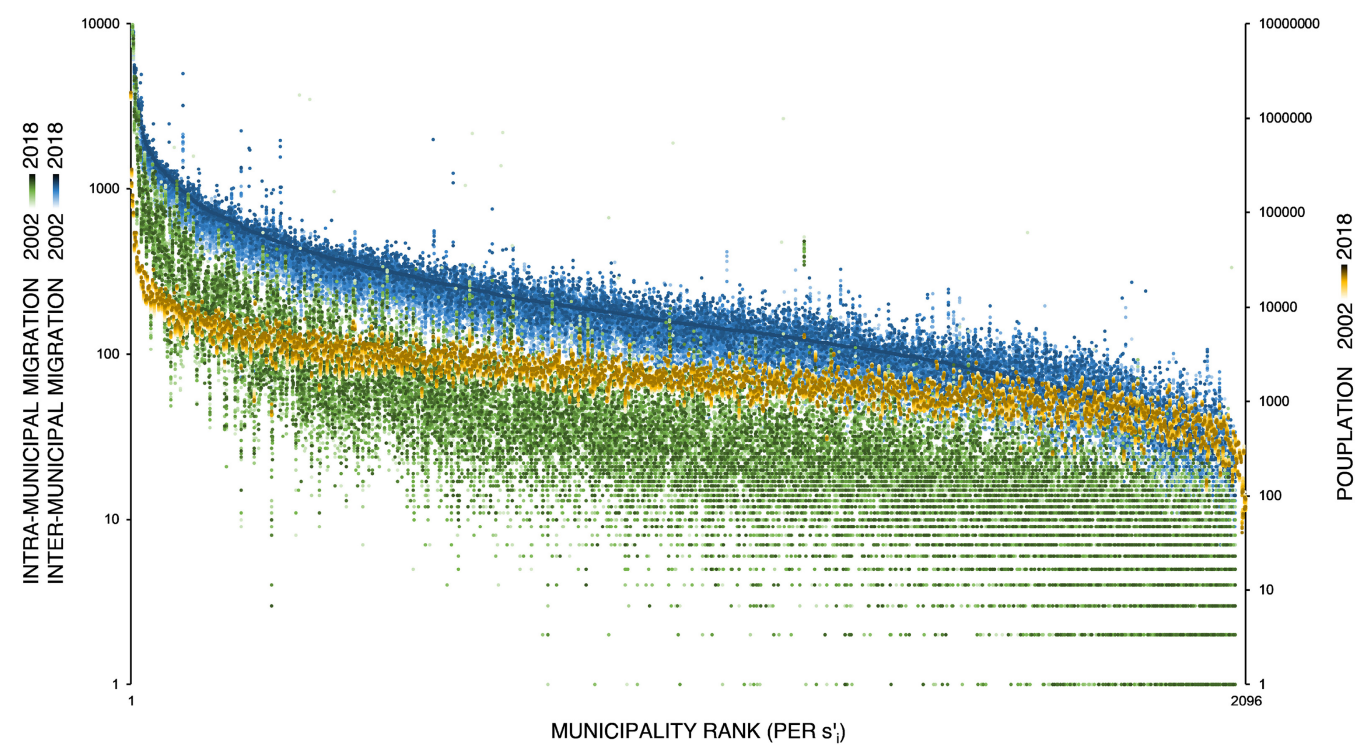

Fig. 2. Distributions of population, intra-municipal, and inter-municipal migration sizes, per Austrian municipality, per year of the period from 2002 to 2018. Vertical axes: Migration/population size (number of persons) on a logarithmic scale. Horizontal axis: Municipality rank according to the average size of inter-municipal migration over the period.

migration at the level of municipality is represented by the node strength [58] of the municipalities in the intermunicipal migration subnetworks $\mathcal{G}^{\prime}{ }_{t}$.

The evolution of the Austrian internal migration network can be brought closer by means of network graphsone of the most straightforwardly useful tools of network analysis that provides a comprehensive picture of migration. Figure 3 shows this evolution, with the emphasis on the growth of inter-municipal migration weights. One can observe a robust network structure, where the graphs do not seem to move toward completeness but whose weights are generally growing on a fixed set of links. In other words, in a loose interpretation, about the same migration links are traversed by an increasing number of migrants each year. Self-loops (intra-municipal migrations per municipality) are omitted from this representation to maintain clarity, but considering the aforementioned strong correlations, one can imagine the weights on loops from/to each municipality as being proportional to the weights by which each municipality interacts with other municipalities. Besides the consistent spatially distributed growth of migration, network graphs unveil a high level of reciprocity in the network, as for every major link there is a counter-link of seemingly proportional weight. The preceding descriptions on the "looping" and reciprocal migration are in line with some of the oldest internal migration observations (see the work of Ravenstein [59]).

The correlations among municipal values/value rankings between different yearly records of any of the three observed categories (inter-municipal migration, intra-municipal migration, and population) are extremely strong ( $\min \rho=0.996)$. Very strong correlations also exist between different yearly records of inter-municipal and intramunicipal migration at a link level $(\min \rho=0.960)$. This, along with the network visualizations, warrants the historical validity of findings that we will have obtained from analyzing any particular year. In further analysis, we hence run and interpret network measures and models primarily on the migration network of the year 2018, the most recent period which for data was retrievable at the time of performing these analyses. Also in terms of notation, unless the period of observation is specified, all calculations refer to the year 2018. 


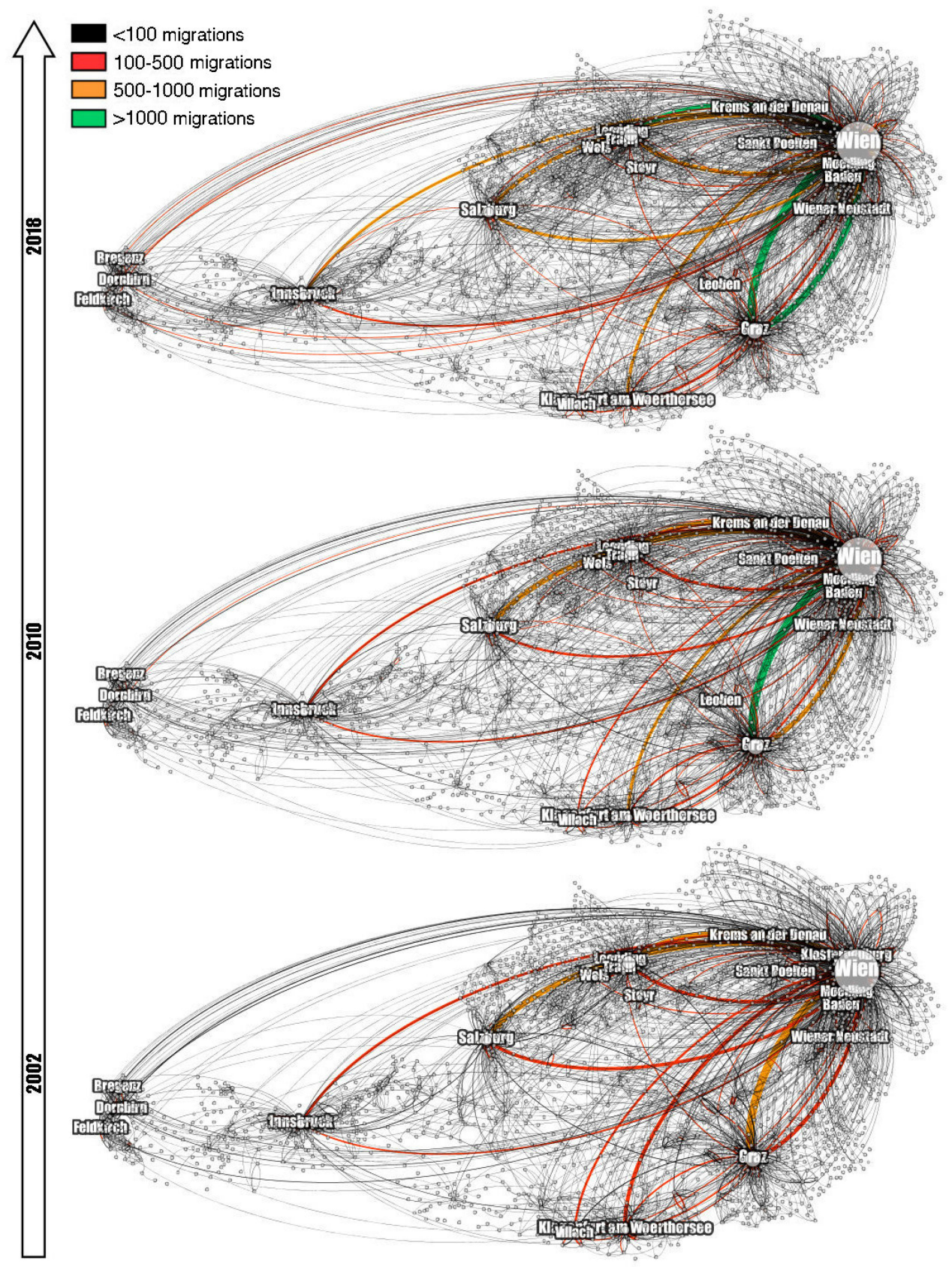

Fig. 3. Evolution of the inter-municipal migration network of Austria from 2002 to 2018. Edges with $w_{i j} j_{t} 10$ migrations are omitted in all graphs for better readability. Labels indicate the 20 most central nodes in each $\mathcal{G}^{\prime}{ }_{t}$, in terms of their node strength $\left(s_{i t}\right)$ and also the basis for size of the labels (nodes). Direction of migration is reflected in the clockwise curvature of links. 


\subsection{Centrality of Municipalities and the Reduction of Spatial and Social Scope}

As described in Section 2, before we initiated this analysis, in our earlier work [16] we assessed the feasibility of application of different network measures and models on a network of human migration, along with appropriate ways of network abstraction from migration data. There, we argue that the reduction of scope to the exclusively inter-nodal connections (the disregard of self-loops typical for network analyses) must be backed up by some sort of a statistical validation for the generalization of findings to the network as a whole. This section is dedicated to the application of node centrality measures for an assessment of municipalities' relative importance, but through the statistical comparisons of these indicators' results after being applied on the complete network versus on the isolated inter-municipal migration network, we are also able to achieve a reduction of the overall complexity of the analysis. Further reductions can be achieved as well in terms of comparisons of results of these indicators' applications for networks and subnetworks based on nationality and gender dichotomies. We turn first to the calculations and comparisons of the most basic centrality measures: node strength and degree centrality.

4.2.1 Node Strength and Degree. The list of strength and degree centrality values for municipalities per all of the abstracted migration (sub)networks is provided in the supplementary material [60]. We point back also to Figure 3, which shows the 20 most central municipalities ranked by node strength in $\mathcal{G}$. For the orientation, note that the value for the strongest node in the network is $\max s_{i}=s_{\text {Wien }}=443,603$, followed by $s_{\text {Graz }}=86,306, s_{\text {Linz }}=51,630, s_{\text {Salzburg }}=36,143$, and $s_{\text {Innsbruck }}=35,799$, and that the value for the weakest node equals 2. The same nodes are at the top in $\mathcal{G}^{\prime}: \max s_{i}{ }^{\prime}=s_{W i e n}{ }^{\prime}=69,935$, followed by $s_{G r a z}{ }^{\prime}=24,790$, $s_{\text {Linz }}{ }^{\prime}=18,540, s_{\text {Salzburg }}{ }^{\prime}=11,567$, and $s_{\text {Innsbruck }}{ }^{\prime}=11,365\left(\min s_{i}{ }^{\prime}=2\right)$. Naturally, as was raised in the previous section, these rankings, especially for the top municipalities, are in strong correlation with population sizes; however, there are notable exceptions where smaller settlements rank over the larger, such as Wiener Neustadt over Sankt Pölten, Feldkirch over Leonding, and Klosterneuerburg over Traiskirchen. These ranking differences are the result of the influence of other migration drivers and deterrents, such as the physical distance to the more populated, as well as to the "popular" areas in terms of job opportunities and so forth. The rankings inform the policymakers about the relative importance of the municipalities, which can serve as a basis for the discussions on the possible factors influencing migration preferences, and whose affecting might modulate the evolution of the phenomenon.

The node strength values can be considered as total migratory activity of a municipality when calculated on the complete network $\left(s_{i}\right.$ in $\left.\mathcal{G}\right)$ or total migratory exchange of a municipality when calculated on the network of exclusively inter-municipal migration $\left(s_{i}^{\prime}\right.$ in $\left.\mathcal{G}^{\prime}\right)$. These two nodal features show to be in very strong correlation: $\rho\left(S, S^{\prime}\right) \approx 0.96$ (Pearson) and $\approx 0.98$ (Spearman). Very strong correlations are also found when comparing relative strength values and value rankings in the complete network versus that of the inter-municipal subnetworks for each group; the smallest correlation of strength values is with non-nationals $\left(\min \rho\left(S_{O}, S_{O}{ }^{\prime}\right) \approx 0.93\right.$ ), although rank correlation for this same group is $\approx 0.98$. Nearly perfect correlations were found between strength values (ranks) of complementary group networks $\left(S_{A}\right.$ vs. $S_{O}, S_{M}$ vs. $\left.S_{F}\right)$. Strength distribution plots provided in Figure 4 visually grasp these high correlations.

All distributions were found to follow a power law with exponents $(\alpha)$ as specified in Figure 4, testing for the power law fit was performed according to Clauset et al. [61], with all $p$ values for the Kolmogorov-Smirnov test calculated well above $0.1\left(\min K S . p=0.504\right.$, for $\left.S_{O}\right)$. Based on the correlation analysis for node strength values and value rankings, captured also by the charts, it shows that both migratory activity and migratory exchange of municipalities are strongly proportional and almost equally intense when we compare migration networks of males with migration networks of females. Migratory exchange in the inter-municipal migration network of nationals versus that of non-nationals shows to be highly proportional as well. For non-nationals, total migratory activity appears to be somewhat less in line with migratory exchange across municipalities, where non-nationals tend to make more intra- than inter-municipal relocations. 

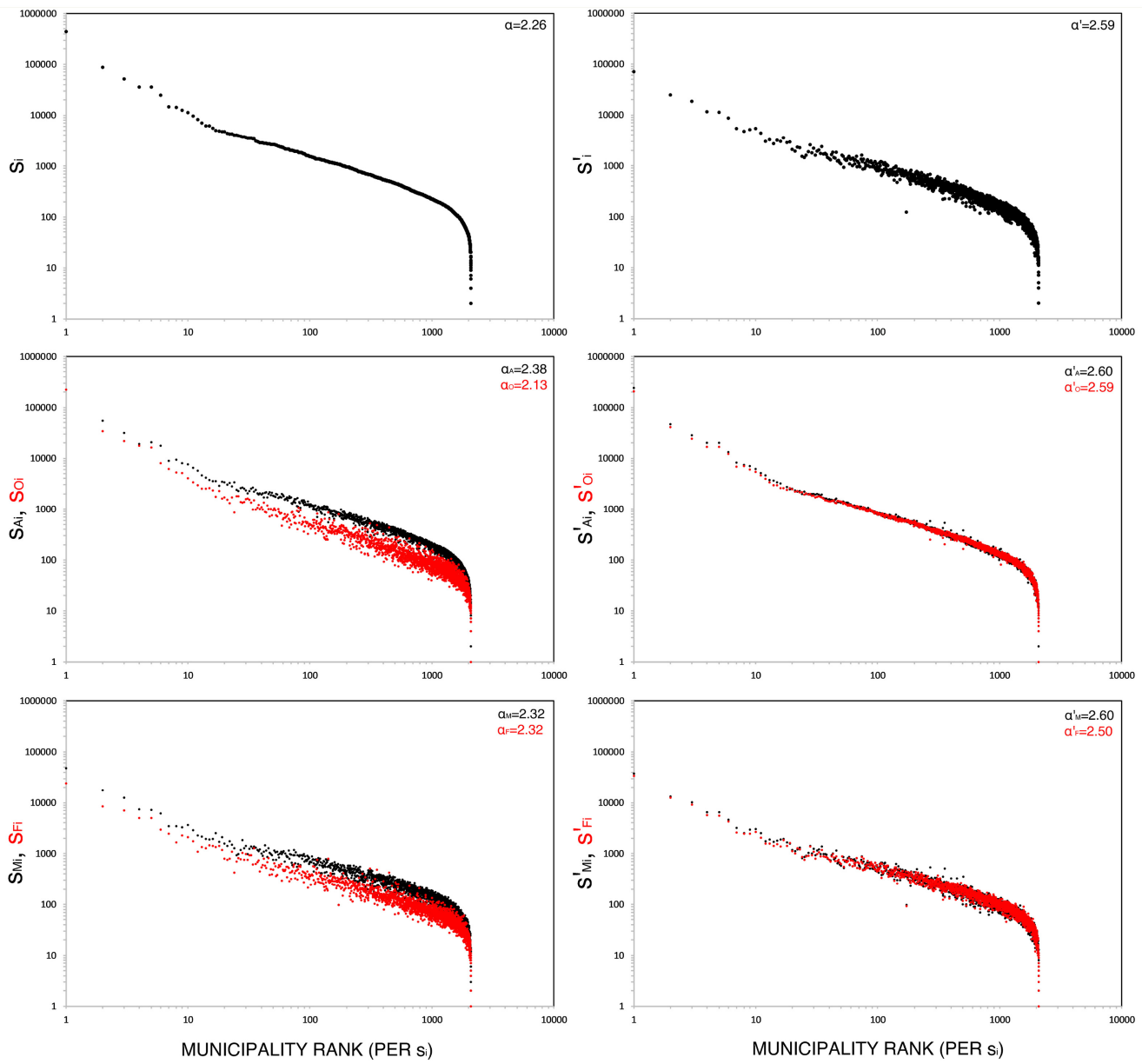

Fig. 4. Node strength distributions. All plots on the left pertain to networks including loops (Gs), and all plots on the right pertain to networks excluding loops $\left(\mathcal{G}^{\prime} \mathrm{s}\right)$. Top: Networks as a wholeCenter: Networks of nationals (in black) versus nonnationals (in red). Bottom: Networks of males (in black) versus females (in red). Logarithmic scale is applied on all axes.

When observing the diversity of location municipalities exchange migration, which is reflected through degree centrality $\left(k_{i}\right)$, we always find a nearly perfect correlation between any two sets of degree values (ranks) of the network and its inter-municipal subnetwork, as well as between and within values (ranks) in networks of nationality/gender groups. For the exact degree values for each municipality, consult the supplementary material [60]. Naturally, degree distribution was also found to follow a power law, with the exponent of 3.28 in $\mathcal{G}$ and 3.25 in $\mathcal{G}^{\prime}$. Virtually identical degree distributions are found between all group networks and their intermunicipal subnetworks. This indicates that both men and women, and nationals and non-nationals, migrate along the same migratory links.

Nearly perfect correlations are also found between direction-respective degree and strength values and ranks: $\rho\left(K^{\text {in }}, K^{\text {out }}\right), \rho\left(S^{\text {in }}, S^{\text {out }}\right), \rho\left(K^{\text {in' }}, K^{\text {out }}\right), \rho\left(S^{\text {in }}, S^{\text {out }}\right) \geq 0.99$. Similarly, nearly perfect correlation is found between respective directional centralities in each group network. This indicates that, in Austria, the more inmigrated settlements are ubiquitously more out-migrated settlements, and points to a high link (weight) reciprocity in the network, which, among other network structural characteristics, we cover in detail in the next section. 
The confirmation on the vast similarities between and across networks and inter-municipal subnetworks legitimizes the reduction of analysis' scope from the full network(s) to the inter-municipal migration subnetwork(s). Moreover, the vast similarities in inter-municipal migration across different groups allow for a generalization of findings obtained from the analysis of the inter-municipal subnetwork as a whole to any of the particular inter-municipal group subnetworks. This is certain in the case of gender groups, since men and women exhibit particularly similar migration tendencies; in other words, what we find for the subnetwork of inter-municipal migration may be considered valid equally to men as to women. Last, from the observations of complementary groups in terms of nationality, although the patterns are again vastly similar, the tendency for more intense intra-municipal migration compared to total migration with non-nationals requires us to be cautious with such generalizations. The cross-group comparisons extend to the following section.

4.2.2 Hubs and Authorities. Migration by definition does not presume indirect connectivity; when a migrant relocates from one municipality to the other (or the same), it is expected that $\mathrm{s} / \mathrm{he}$ will stay at the migrated location for a longer period of time, at least as long as the recording period (1 year). The network is not formed by overlapping paths but by one-time movements of "batches" of people from one place to the other. Even if the network would be based on the data of finest granularity, of every single individual relocation, the network of any particular period would still be formed by bilateral one-time movements that start at one municipality and sink in the directly connected other. Thus, the connectedness of one location does not effectively relate to the connectedness of the other. Migration is, moreover, relatively independent of the physical infrastructure that links municipalities, and much less dependent than other mobility networks, such as the network of commuting, which directly depends on the transportation infrastructure. Given these specifics, the selection and interpretation of metrics that incorporate neighbors-of-neighbors connectedness is not straightforward. We elaborate more on this in our related work [16].

In our application, we turn to the more complex centrality indicators, which take into account directedness of links, PageRank centrality $(P R)$ [62], and Hub $(H)$ and Authority $(A)$ centrality (the HITS algorithm) [63]. The two measures developed for the binary directed World Wide Web have been updated to suit the weighted directed network observation in numerous applications (e.g., Deguchi et al. [64] and Bartholdi et al. [65], to just name a few). In our application, we will follow the common practice of weight normalization, $\frac{w_{j i}}{\sum_{k} w_{j k}}$, where $w_{j i}$ is the edge weight from municipality $j$ to $i$. The Hub, Authority, and PageRank scores for all municipalities are available in the supplementary material [60].

Via the application of the HITS algorithm, municipalities get assigned higher in-migration scores (Authority scores) if they receive more migrants from municipalities with higher out-migration scores (Hub scores) and vice versa. With regard to PageRank, its values theoretically represent the likelihood of a particular municipality being the chosen migrated destination of a "random" migrant that joined into the network at any given moment, based on the entire structure of the network (direct and indirect connectivity). Both of these measures, especially the latter, may be useful for migration forecasting. For example, the policymakers could use these indicators to estimate the distribution of larger migration flows coming into the country across its municipalities. However, as much as these same measures get continually updated by additional variables against their original formulation to be optimized for their original application (ranking Web pages), so does migration forecasting require complementing these indicators with numerous variables that stand as drivers of human migration to get to the optimal probabilistic algorithm. These algorithms' rankings nevertheless are, same as strength and degree rankings, a good starting point for policymakers' discussions on possible migration factors, and their influencing with the purpose of controlling the migration flows.

We can use the raw results of these algorithms for making additional cross-group (nationals vs. non-nationals, male vs. female) comparisons. As these indicators encapsulate the whole network structure, they can be used to re-evaluate the appropriateness of generalizations we proposed in the previous section. As we did for node strengths and degrees, here we also sought for correlations between Hub, Authority, and PageRank values (ranks)

Digital Government: Research and Practice, Vol. 2, No. 3, Article 25. Publication date: July 2021. 

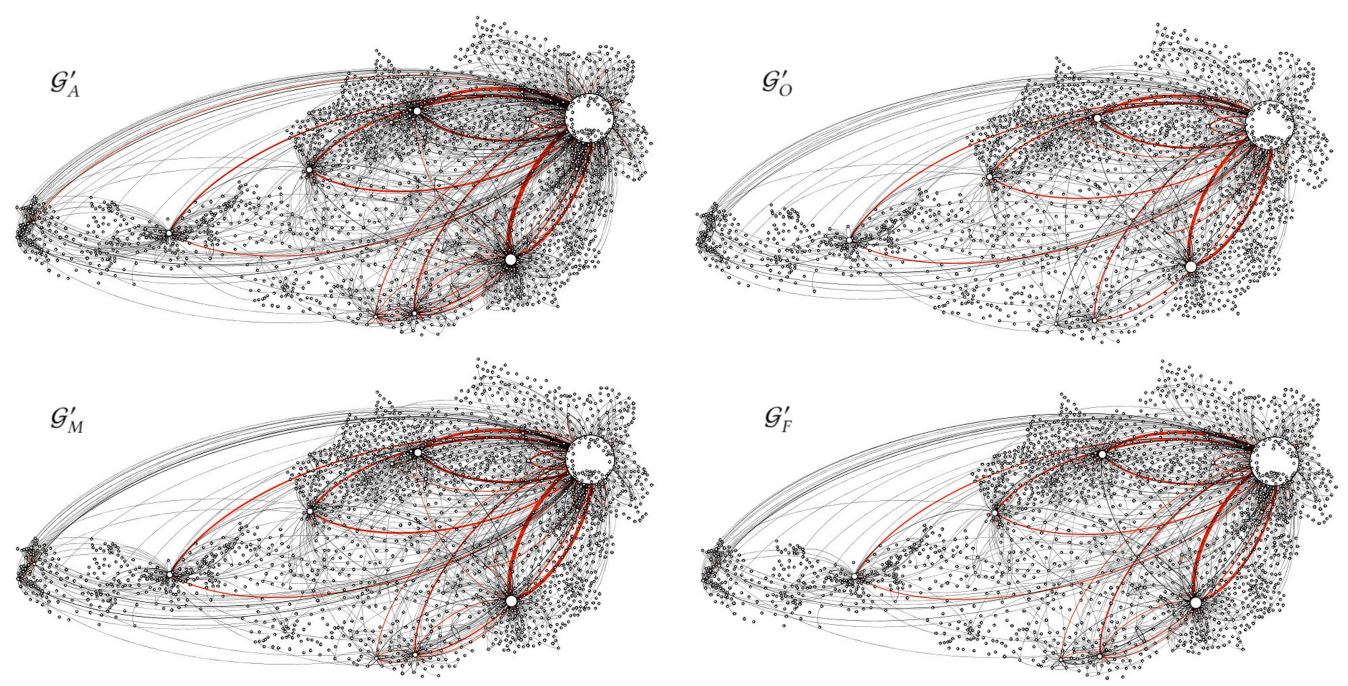

Fig. 5. Austrian inter-municipal migration nationality and gender subnetworks in 2018. Edges with $w_{i j} \geq 100$ migrations are marked in red, and edges with $w_{i j} \leq 10$ migrations are removed in all graphs. Links thickness is proportional to link weights $\left(\max w_{i j}{ }^{\prime}=w_{\text {Graz, }}\right.$ Wien ${ }^{\prime}$ in all four networks; $\left.\max w_{i j_{A}}{ }^{\prime}=922, \max w_{i j_{O}}{ }^{\prime}=659, \max w_{i j_{M}}{ }^{\prime}=827, \max w_{i j_{F}}{ }^{\prime}=754\right)$. Direction of migration is reflected in the clockwise curvature of links.

calculated in migration subnetworks of nationals, non-nationals, and males and females. We found strong correlations here as well: all correlations were above 0.97 , except $\rho\left(A_{A}^{\prime}, A^{\prime}{ }_{O} \approx 0.85\right)$. One more network graph visualization is provided in Figure 5 to elucidate how greatly similar network patterns are among the examined groups.

In conclusion, we establish that the discovered similarities in the structure of the four groups through direct and indirect connectivity grant us to generalize the findings derived for the total inter-municipal migration subnetwork $\left(\mathcal{G}^{\prime}\right)$ to any of the inter-municipal group subnetworks $\left(\mathcal{G}_{A}^{\prime}, \mathcal{G}_{O}^{\prime}, \mathcal{G}_{M}^{\prime}\right.$ and $\left.\mathcal{G}_{F}^{\prime}\right)$. Combined with conclusions from the previous analysis of direct connectivity, the findings from the analysis of the inter-municipal migration subnetwork $\left(\mathcal{G}^{\prime}\right)$ may be generalized to the network $(\mathcal{G})$ as a whole. Henceforth, we will continue the analysis on the inter-municipal migration subnetwork only $\left(\mathcal{G}^{\prime}\right)$, and in the descriptions and interpretations we will refer to the same as just "network" for simplicity. We also offer an easy-to-use, interactive visualization of this network at https://flowmap.blue/1zA3XV4fYshsdkwmWtfixNt5pVBtco4NPAjcUe-FtAoc.

\subsection{Network Structure}

In the previous section, we touched upon some of the characteristic structural features of the migration network when discussing the application of eigen-centrality measures. In this section, we further analyze its structure using the network structural metrics refined in our earlier work [16].

4.3.1 Reciprocity. Perhaps the most prominent feature of the Austrian inter-municipal migration network is reciprocity. This reciprocity was indicated by strong correlations between outward and inward municipality centrality values, and is apparent in network visualizations (Figure 3). For this part of the analysis, we return to incorporate the temporal aspect of network development, as by observing the temporal reciprocity we can grasp the extent by which inter-municipal migration systematically contributes to the population size of municipalities. In addition, we can once again validate the reduction of temporal scope by re-examining consistency in network structure. 


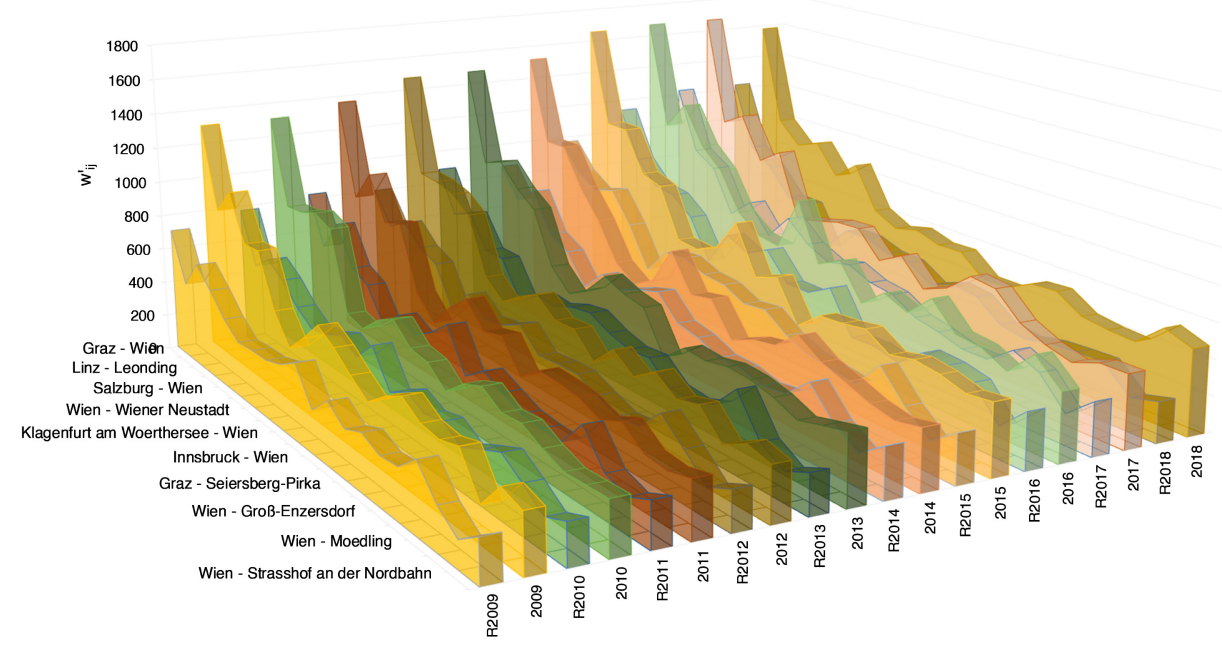

Fig. 6. Reciprocated component of the top 20 migratory links from 2009 to 2018. Top migratory links (counter-links) in terms of their average weights are shown over the mentioned period. The reciprocated component for each respective year is designated by the prefix "R." Vertical axis: Link migration weights.

The principles by which to measure reciprocity in weighted networks have been debated. These issues are raised in the work of Squartini et al. [66], whose definition and terminology we will use in this demonstration. Among the problems are how to handle the absence of reciprocation $\left(w_{j i_{t}}=0\right)$, and how to handle situations where $w_{j i} t<w_{i j_{t}}$. For a general evaluation, we respect both of the preceding cases and consider the total reciprocated component in total migration over the whole analyzed period (2002-2018): $r=\frac{\sum_{i} \sum_{j \neq i} w_{i j}^{\leftrightarrow \prime}}{\sum_{i} \sum_{j \neq i} w_{i j}^{\prime}} \approx 0.61$. This value, of about 0.60 , remains almost unchanged when calculated for the networks of each particular year ( $\left.\min r=r_{2005}=0.59, \max r=r_{2017}=0.61\right)$, which is another confirmation of the historical validity of results obtained from analyzing a 1 (last) year's migration network. In a loose interpretation, in Austria, for every 100 people relocating from an origin to a destination during a particular year, there will be on average 60 people relocating from that same destination to the same origin during that same year.

Naturally, reciprocity is higher between municipalities of higher centrality ranks. In Figure 6, we provide a glance at the general reciprocity pattern, on the example of the 20 most central links according to the average migration (including counter-migration) over the 10-year period from 2009 to 2018). Note that the top 3,888 migratory links and their counter-links (note that $\max \sum l_{i j^{\prime}}{ }^{\prime}=\sum l_{i j}{ }^{\prime}{ }_{2018}=58428$ ) take up more than $50 \%$ of migratory activity of that period. The precise specification of these top links, their counter-links, and weights is provided in the supplementary material [60]. By this specification, the policymaker is informed about the relative importance of links, as complementary to the relative importance of nodes covered in the previous section, which aids discussions on potential migration factors working on particular bilateral ties, and how to affect these factors.

With such intense and stable reciprocity, it can be expected that the non-reciprocated component of migration flow goes systematically in favor of some, particularly large, municipalities. The share of migration that spills over, on a yearly basis, in favor of larger municipalities, can be traced by calculating the difference in total migration that goes from less populated to more populated municipalities and vice versa (Figure 7). The average spillover over the investigated period is $2.08 \%$, which can be considered as the average contribution to urbanization coming specifically from internal migration. A further investigation that can be undertaken by the policymakers directly using the supplementary material [60], particularly observing the non-reciprocated 


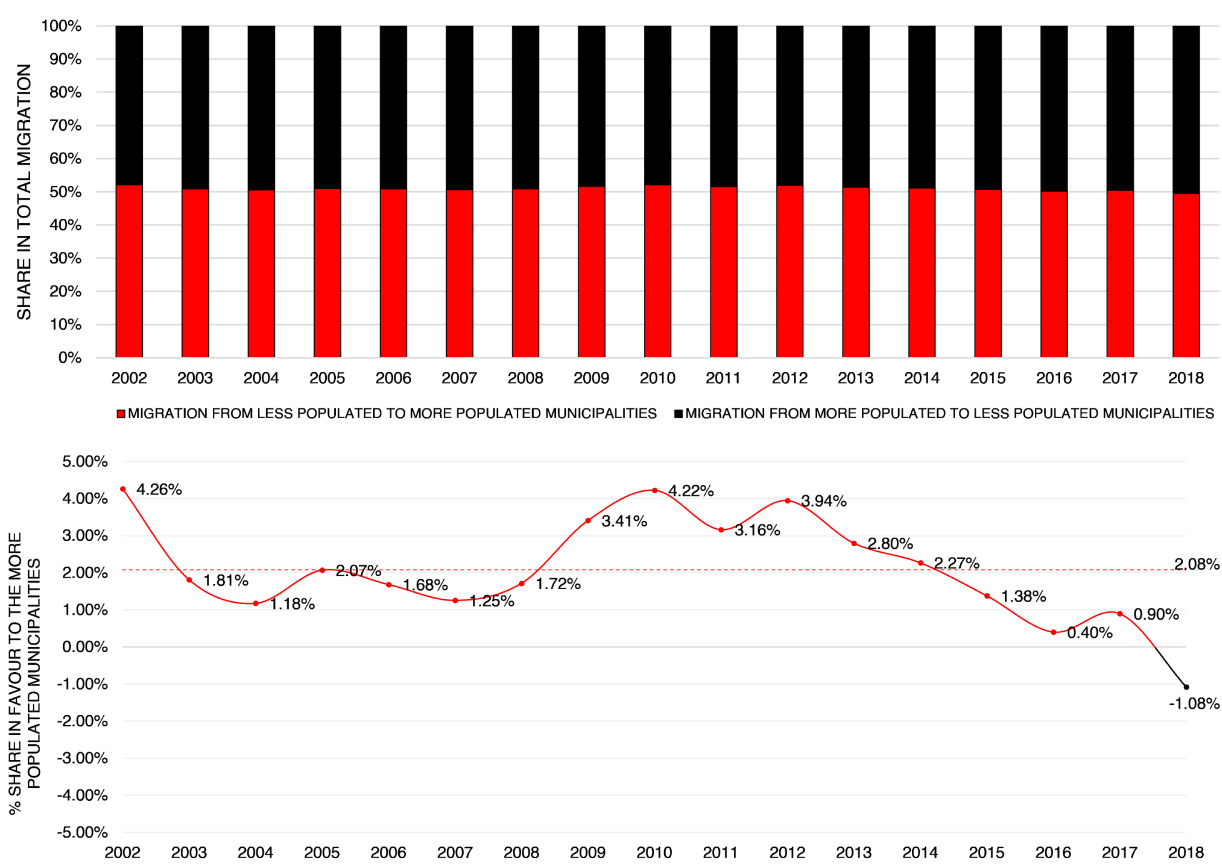

Fig. 7. Migration's contribution to urbanization, 2002-2018. Top: yearly ratio of total size of migration from less-to-more vs. from more-to-less populated municipalities. Bottom: yearly incremental shares of total migration spilling over in favour of the more populated municipalities.

component per each inter-municipal link and adjacent municipalities, can show which exact cities are growing faster relative to the other, further aiding the discussion on factors of this growth.

4.3.2 Assortativity and Transitivity. The amount of network reciprocity established in the previous section appears to interfere with the notion of network assortativity, whether more migrated tend to inter-migrate more with more migrated, or with less migrated municipalities. This occurs because, following the original assortativity definition [67], the remaining (weighted) degrees of the two linked municipalities overlap to large extent. The weighted assortativity coefficient (adjusted for the weighted directed application as in the work of Csárdi and Nepusz [68]), calculated at -0.05, would suggest that we can conclude-at best-that the network moves negligibly toward the disassortative side. Otherwise, we cannot quantify the tendency of settlements to inter-migrate with high or low migrating neighbors. The canceling-out effect is clear when comparing each particular municipality's strength and its weighted average nearest neighbor degree (WANND) [58], as presented in Figure 8 on the left.

The highly reciprocal network structure interferes with the notion of transitivity as well. Figure 8 , on the right, shows the virtually uniform distribution of the values for the weighted (local) clustering coefficient (WLCC) [58] value for municipalities in $\mathcal{G}^{\prime}$. This means that intensity of migration interaction of each municipality with two adjacent municipalities is always in proportion to the intensity and diversity of its total in-/out- migration. This is reasonable, as the two adjacent municipalities observed return a significant amount of migration flow, simultaneously, to the one being evaluated.

The preceding two network-structural measures were among the most extensively applied measures in former migration studies, although these were mainly applied in their nascent, binary form [16]. What we theorized as potentially reducing the interpretability of these, and structural metrics in general (when applied on the 

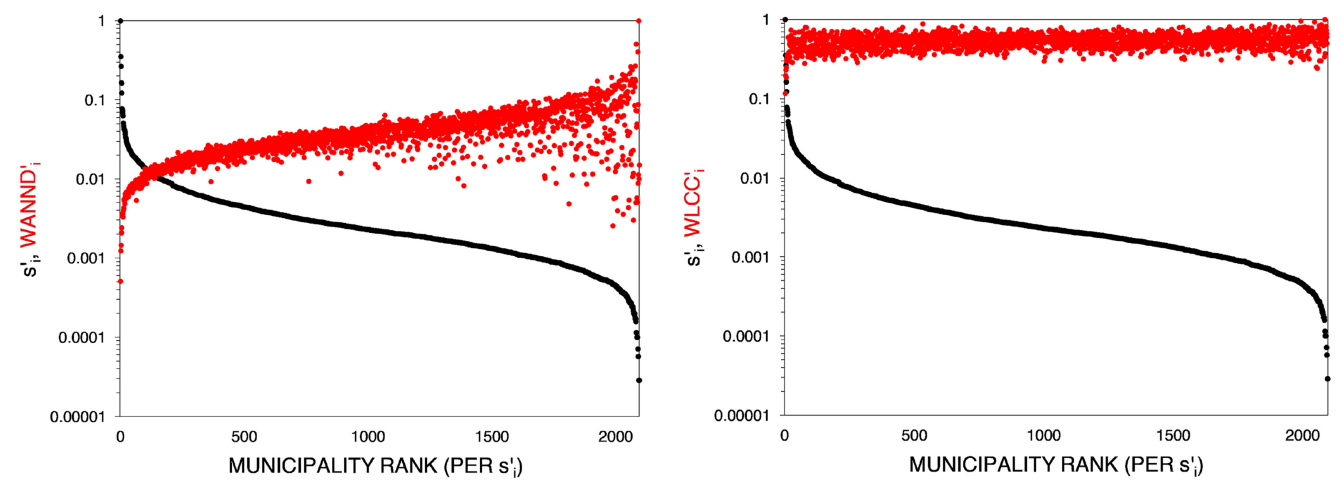

Fig. 8. Assortativity and transitivity indicator distributions. Left: Distribution of WANND (in red) against the distribution of node strength (in black). Right: WLCC (in red) against the distribution of node strength (in black). WANND values rescaled by maximum. Value (vertical) axes of a logarithmic scale.

migration network in the most realistic abstraction), is the non-existence of network paths-a problem we discussed while analyzing the eigenvalue centralities in the previous section. By this application, we demonstrated how the pronounced trait of high reciprocity in the migration network is the primary cause for the lower interpretability of some typical structural indicators. This may serve as an invitation for the network theorists to find ways to advance the network metrics to account for high reciprocity, which may not be the trait exclusively present in the network(s) of human migration.

4.3.3 Modularity and Random Migrant Clustering. To understand the community structures in the network, we have utilized a couple of methods based on optimization of modularity [69] and the InfoMap algorithm [70] based on the description of random walk through the network. The modularity optimization methods include the Louvain algorithm [71] and the Fastgreedy algorithm by Clauset et al. [73]. Since highly reciprocal migration was found to be consistent across municipal pairs, we considered $\mathcal{G}^{\prime}$ as undirected, as required for the application. Both modularity optimization algorithms detected the same, highly modular structure $(Q=0.68)$, with 9 communities. InfoMap, which was straightaway applicable, detected a more fine-grained modular structure with 47 communities. Figure 9 shows the two sets of communities obtained by using the two methods, whereas the community membership allocation per different algorithm is provided in the supplementary material [60].

Both of the approaches-modularity optimization and random walk optimization-provide reasonable results. The first two algorithms identify relatively large modules centered at the largest Austrian cities that exchange most of migration with surrounding settlements. InfoMap traces smaller municipal clusters in which, according to the suggested theoretical interpretation, a random migrant spends a longer period of time before departing for another cluster. These clusters are obviously determined by geographical proximity as well, although some influential cities stretch into more remote areas, such as Klagenfurt stretching wide in the southern part of the country or Graz stretching inland and toward Vienna.

The policymakers profit from these results, as they can match the current administrative subdivision with the hypothetical subdivision based on migration, as one of the most important factors in state organization. The same as with node and link relevance rankings covered in previous sections, the information on different community structures can serve as input for understanding migration factors working in different urban-centered regions.

\subsection{Network Models}

At the end of our analysis, we implement two link weight estimation models, which require a minimum of network-external variables for the purpose of forecasting. Both models are known to work well in explaining 

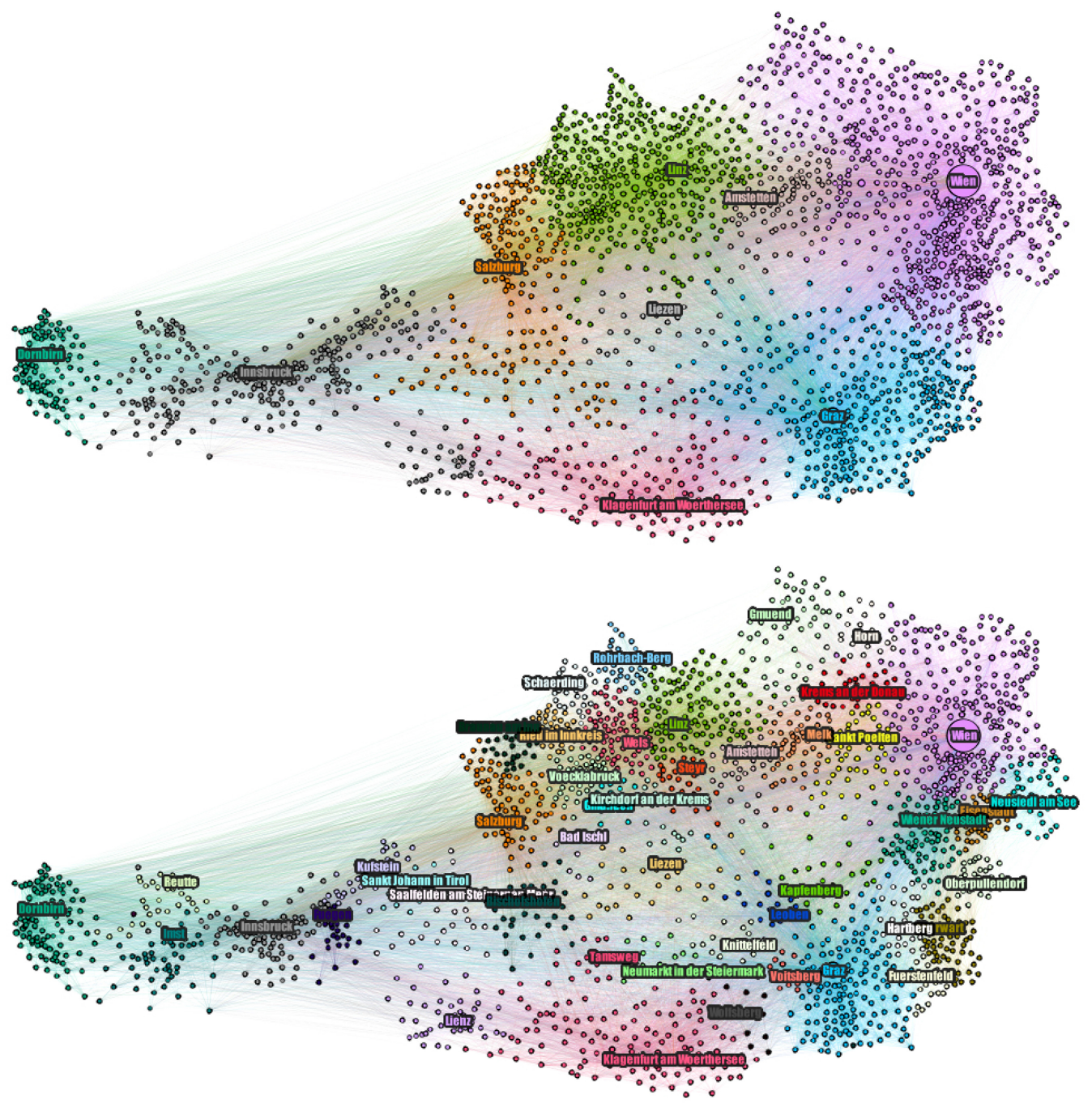

Fig. 9. Community structures of internal migration network of Austria. Top: communities detected by Louvain/Fastgreedy algorithm modularity optimization algorithm. Bottom: communities detected using InfoMap minimal random walk description code length. Labeled are the municipalities from each community with highest node strength.

migration and mobility within other countries or internationally, and have been used extensively in other network studies of migration [16]. Shared variables for both models are defined as follows: $p_{i}$ refer to the populations of origin and destination municipality on January 1, 2018, respectively (data obtained from Statistics Austria), and $d_{i j}$ are the great-circle distances between municipal centers of origin-destination municipality pairs. Both models are tested for the 1,000 links carrying the highest weights.

4.4.1 Gravity Law Model. We first tested the hold of the gravity law [74], where we produced estimated weights as $\hat{w}_{i j}{ }^{\prime}=p_{i}^{\alpha} p_{j}^{\beta} d_{i j}{ }^{-\gamma}$. Best fit, in terms of the maximum coefficient of determination in a linear regression on the two variables, was found for $\alpha \approx 0.33, \beta \approx 0.31$, and $\gamma \approx 0.29$. Real and model-estimated values, as well as the linear regression estimation, are provided in Figure 10 (left). Exact values for all variables in the model 

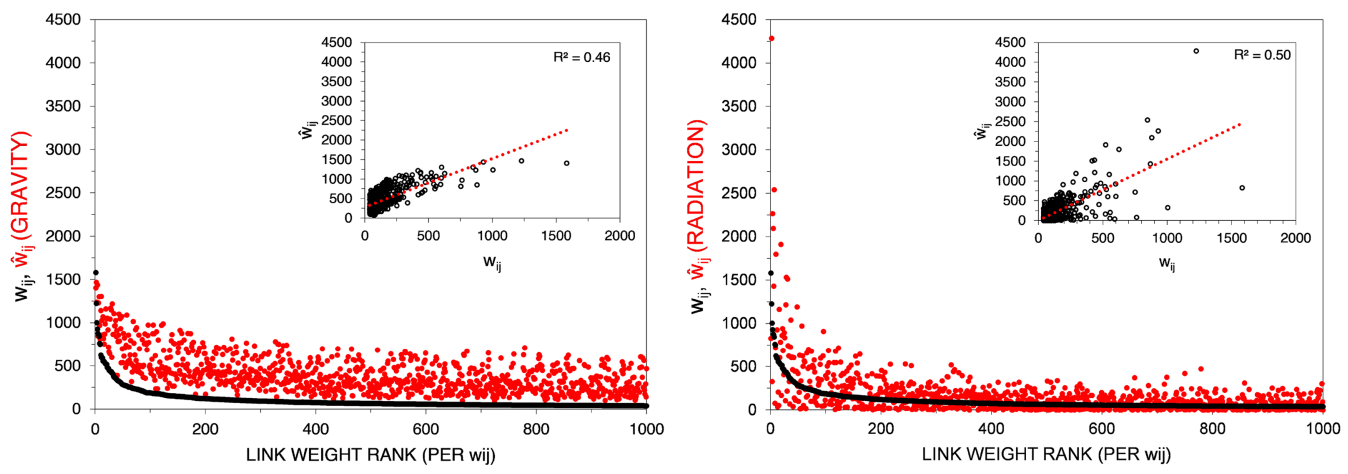

Fig. 10. Gravity and Radiation model estimations. Left: Gravity Law model-estimated values (in red) vs. real values (in black) of the 1000 links of highest weight in $\mathcal{G}^{\prime}$. Right: Radiation model-estimated values (in red) vs. real values (in black) of the 1000 links of highest weight in $\mathcal{G}^{\prime}$. In top right of both charts: simple regression plots for real vs. model-estimated values.

are available in the supplementary material [60]. As expected, the gravity law equation produces estimated values that are in good proportion with actual migrations. However, it is also clear that the model systematically exaggerates flow predictions, which is not unusual (e.g., see the work of Kaluza et al. [75]).

4.4.2 Radiation Model. An extension to the gravity model that was offered to correct for its biases has been proposed by Simini et al. [76]. The main notion behind the model is that employment opportunities sought for by potential migrants are in proportion to the size of the population within the radius of a circle, $s_{i j}$, of which the circle is centered at $i$ and tangent to the center of $j$, excluding $p_{i}$ and $p_{j}$. By that notion, it is not only the gravity of the estimated adjacent municipality that is taken into account in the prediction but also the gravity of all locations surrounding the migrant-sending location. Exact values of all components of the model are available in the supplementary material [60], as well as within-radius municipalities for each evaluated link. Note that on some occasions, from the top-1,000 links between municipalities that were in immediate proximity, within-radius population $\left(s_{i j}\right)$ would return a zero value. These links are excluded from the estimation of both the Gravity and Radiation models. Model-estimated weights were derived following the original model as $\hat{w}_{i j}{ }^{\prime}=M_{i} \frac{p_{i} p_{j}}{\left(p_{i}+s_{i j}\right)\left(m_{i}+p_{j}+s_{i j}\right)}$, where $M_{i}=p_{i} \frac{\sum_{j} w_{i j}{ }^{\prime}}{\sum_{i} p_{i}}$. The Radiation model shows to be much more balanced in its predictions, which is observable from Figure 10 (right), and the policymakers are therefore recommended to use the Radiation model for migration forecasting purposes.

It is clear, however, that the two models are not sufficient in explaining migration. The Radiation model has somewhat better explanatory power, yet both models are able to explain only about half of the variance of real migration (see $R^{2}$ values in Figure 10). This is not odd, as the number of variables identified by migration scholars as being relevant drivers or deterrents of migration is vast-from economic, such as income, (un)employment, or living expenses, over socio-cultural such as education, lingual, or religious proximity, to external such as climate or natural resources. Still, it is impressive how useful these two natural variables are for predicting migration, which are now also shown for the case of Austrian internal migration.

\section{FINDINGS}

In this section, we briefly recapitulate our analysis' main findings. For the consequences of these findings and the policy contributions, the reader should consult Section 4 to obtain a detailed view, or Section 6 where we shortlist the main contributions of each indicator and model application.

The overall size of internal migration in Austria has been steadily increasing from about $8 \%$ in the starting year of observation (2002) to about $9 \%$ in the ending year of observation (2018). In any year of observation, 
more than half of relocations in Austria (55\% on average) are relocations within one and the same municipality (intra-municipal migrations); however, there is a higher increase of inter-municipal migration relative to intra-municipal migration and to population. The relative proportions of these three categories (population, inter-municipal migration, and intra-municipal migration) are consistent at the level of individual municipalities considering each year's distribution over the whole analyzed period. One can observe a robust network structure, where migration network in time does not seem to move toward completeness but in which migration weights are generally growing on a fixed set of links. Temporal validity has been established for the case of observing one (last) period only: 2018.

Node centrality indicators, namely node strength, degree centrality, PageRank, and HITS algorithm centrality have been calculated for the discrete last year's period, and are provided in the supplementary material [60]. These measures show relative attractiveness or repulsiveness in terms of the different direction of the indicators, which is reported alongside the composite indicator values. PageRank and HITS algorithm centrality deliver the basic probabilities of migration being distributed over each municipality in the network when not taking into account the different migration factors, but only the inherent network structure. The preceding set of node centrality indicators is calculated and reported for the inter-municipal subnetwork, as well as for the total and inter-municipal subnetworks of Austrian nationals, non-national, and males and females.

Correlation analysis of these indicator values and ranks applied to various subnetwork reductions validated the spatial and social reduction of scope-that is, observing inter-municipal movement only-without regarding any migrant groups separately, as we show that both men and women, and nationals and non-nationals, migrate along the same migratory links with consistent migration intensity. Although the patterns are vastly similar across all groups, the tendency we observed with non-nationals for more intense intra-municipal migration compared to global migration and the rest of the groups requires caution with generalization on the findings concerning that particular group.

The most prominent global structural trait of the network is its reciprocity; the total reciprocated component in total migration, over the last 10 years observed (2009-2018), as well as within each particular period of the whole period observed (reconfirming temporal validity), is about 60\%. The top nearly 3,900 migratory links and their counter-links, from the total of nearly 59,000 links, take up more than $50 \%$ of migratory activity of last 10 years observed (2009-2018). The specification of these top links, their counter-links, and weights is provided in the supplementary material [60]. The share of migration that spills over, on a yearly basis, in favor of larger municipalities (i.e. the migration's contribution to urbanization), is calculated at $2.08 \%$ on average over the whole period observed.

Very high reciprocity in the network affects the sensibility of interpretation of assortativity and transitivity metrics. Ultimately, the network is characterized by neither assortative nor dissasortative mixing; small and large municipalities exchange migration consistently and simultaneously with both small and large municipalities.

Separate clusters that form the network are established using three different community detection algorithms, consequently with two different network visualizations. One is more coarse grained and shows large modules centered at largest Austrian cities. The other is more fine grained, with about 50 smaller clusters. The exact municipality allocation per different algorithm is specified in the supplementary material [60].

The two link weight estimation models-the Gravity Law and Radiation models-are both very efficient in forecasting migration. The Radiation model results are, nevertheless, more aligned with the actual migrations, whereas the Gravity Law model systematically exaggerates flow predictions. Exact values for all variables in the model are available in the supplementary material [60].

\section{CONCLUSION AND PROSPECTS FOR FUTURE RESEARCH}

Migration, mobility, and urbanization are interdependent phenomena consequential for global sustainable development. An integrative approach, based on accurate data and reliable descriptive and forecasting models, is 
preferred to analyze these phenomena. People's migration and mobility flows, directly impacting urban growth, make a network structure, with large cities acting as hubs and smaller settlements as spokes. The essential method by which these phenomena can be analyzed comprehensively is network analysis, but the emergent methodology of network science has not yet settled. Very few network analyses have been performed on migration, especially on country-internal migration for which there is accurate and structured data on migration flows. Those that have been performed have not scrutinized the network indicators and models and network abstractions to deliver the most realistic and practically useful results. No such analysis, to the best of our knowledge, has yet been performed for any European country, although Europe, due to convergence of the structures of countries' political and judicial systems, is a very suitable research ground, where conjoining analyses of internal migrations could elucidate migration in the region as a whole. Such knowledge, built on accurate and detailed data, and comprehensive processing and representation tools, is essential in creating coherent and efficient migration policies.

In this context, we underlined the three main aspects as the desired contributions of our study. First, we aimed to contribute to the capacity building regarding the analysis of internal (national) migration data. We did this by providing a systematically derived set of indicators, models, and visualizations, which we tested and argued for in terms of applicability and interpretability in the case of the network analysis of human migration. The systematically derived set of network measures and models applied to the thoroughly elaborated network abstraction and its reductions ensured that insights are realistic and reliable, which is particularly important from the perspective of the policymakers.

Second, we aimed at contributing to the understanding of the shape and scale of the phenomenon of internal migration, particularly toward urbanization and mobility flows between human settlements (i.e., cities, towns, and villages). A network analysis based on a realistic network abstraction enables the policymakers to get a straightforward insight on the holistic network structure, as well as a fine-grained view on the relative attractiveness of particular settlements and migration links. The analysis informs on particular network modules or the spatial range of influence of particular urban centers, thus reducing the complexity for migration and urban growth planning.

Third, we demonstrated our approach on the example of internal migration in Austria analyzed on the level of human settlements and provided a longitudinal analysis for the period from 2002 to 2018. In this application, we have obtained the following main findings.

Internal migration in Austria is shown to increase, slowly but consistently, relative to population, based on the overview of the past two decades. More than half of total internal migrations are always migrations within one and the same location (intra-municipal migration), although inter-municipal migration is rising at a higher pace. The overall network structure is consistent, with migration weights increasing on a generally fixed set of links, which warrants the reduction of temporal scope of the analysis. The relative importance of municipalities is evaluated in a centrality analysis, namely using node strength, degree, PageRank, and the HITS algorithm. The indicators indicate the attractive and repulsive potentials of municipalities, which serves as a good starting point for identifying the various migration drivers and deterrents that have prevalent effect within particular settlements. Besides the municipality relevance ranking, these are used to trace significant similarities between total and separately inter-municipal migration, as well as inter-municipal migration of separately Austrian nationals, non-nationals, and males and females. Methodical application of these indicators ensures reduction of complexity and generalizability of conclusions exclusively based on the inter-municipal network. Inter-municipal migration is highly reciprocal, with a small but persistent contribution of migration in favor of the more populated areas. In addition to the calculation of the effect of migration in terms of urbanization, reciprocity analysis provides insights on the relative link importance, which again serves as a good starting point for identifying the various migration drivers and deterrents working on particular links. The network structure is highly modular, as confirmed by the application of three different community detection algorithms. The communities encapsulate the spatial range of influence of the core urban centers, which aids discussions on potential factors working in 
specific urban regions and generally the state organization planning. Application of the Gravity and Radiation models exhibit strong predictive performance, whereas the Radiation model is more effective and commendable for future utilization in migration forecasting.

This applied network analysis, which explains in detail the process of network abstractions and reductions from basic data, and the selection and the application of network measures, indicators, and visualizations, is offered as means to improve national capacity to analyze and manage migration data. This way of analysis of migration is suggested as an embedded national policy effort on managing migration data more effectively, as is called for by the global development experts. The method and applications designed to be executed on the scale of human settlements are easily replicable to other countries and help policymakers get an overview of human mobility in general, with urbanization as its straightforward consequence. In addition to supporting the policy, our analysis reveals methodological issues that hinder a straightforward application of the network analysis as information for network theorists on where to improve the tools and models.

There are many ways in which this analysis may be advanced. A confirmation of broader validity, as well as usability of the analysis, should primarily be looked for in a comparative analysis with another European country to identify the similarities in migration patterns in the two countries and set out the agenda for eventual joint network analysis for multiple countries with the incorporation of international migration data. Validity and usability of the network approach should also be traced at a narrower level (i.e., intra-settlement migrations). By replicating the analysis on a large city in Austria, such as Vienna for which data are already available, we would be able to confirm whether migration "behaves" similarly at a smaller scale. For these tests for spatial consistency, updates and extensions should be prepared on different indicators and models, making them more abundant. An extended view should be made into the most prominent characteristics of the network, most importantly reciprocity. Where data allows it, deep scans into the temporal development of the networks should be performed, particularly to fully grasp the role of internal migration in urbanization. We have quantified on the example of Austria, roughly, how much migration spills over to the more populated areas. However, it is important to quantify exactly the rate of growth or decline of particular municipalities over the others, or at least per different municipality-size brackets. The calculated rate of contribution to urbanization should be combined with other contributors to urban growth such as natality and international migration.

Moreover, the identified methodological issues such as the lack of appropriate weight adjustments for some network measures, or the interference of reciprocity with other network structural metrics, require a deeper look into the more suitable means to investigate specific networks such as the network of migration. Combined with analyzing the actual policy demands, it is important to advance or create more interpretable and usable metrics. It is imperative to complement migration probability measures such as PageRank, or link weight prediction models such as the Radiation model with data on various migration drivers, to increase their predictive power. All of the important drivers, from those demographic such as age or marital status to those economic such as income or employment, which are, as opposed to nationality and gender, currently not tied in the migration patterns scheme, can be brought in relation to migration through various sorts of modeling. This includes classical econometric-type models, where inter-municipal migrant flows are explained by migration driver indicator values tied to origin and destination municipalities but should also include exponential random graph models, as well as more heuristic models. In the future, we plan to undertake as many of the aforementioned research actions.

\section{REFERENCES}

[1] N. Reslow. 2019. EU External migration policy: Taking stock and looking forward. Global Affairs 5, 3 (2019), $273-278$. DOI : https://doi.org/10.1080/23340460.2019.1604071

[2] United Nations. 2019. SDG Indicators. Retrieved October 8, 2020 from https://unstats.un.org/sdgs/indicators/indicators-list/.

[3] N. Piper. 2017. Migration and the SDGs. Global Social Policy 17 (2017), 231-238. DOI: https://doi.org/10.1177/1468018117703443 
[4] United Nations General Assembly. 2016. Resolution Adopted by the General Assembly on 19 September 2016. Retrieved October 8, 2020 from https://www.un.org/en/ga/search/view_doc.asp?symbol=A/RES/71/1.

[5] E Ordaz. 2019. The SDGs indicators: A challenging task for the international statistical. Global Policy 10, S1 (2019), 141-143. DOI : https: //doi.org/10.1111/1758-5899.12631

[6] F. Laczko. 2016. Improving Data on International Migration and Development: Towards a Global Action Plan. Retrieved October 8, 2020 from https://gmdac.iom.int/sites/default/files/papers/Improving\%20Data\%20on\%20International\%20Migration\%20and\% 20Development-\%20Towards\%20a\%20Global\%20Action\%20Plan\%3F.pdf.

[7] H. De Haas. 2014. Human Migration: Myths, Hysteria and Facts. Retrieved October 8, 2020 from https://heindehaas.files.wordpress. com/2016/02/de-haas-2013-wolfson-human-migration-myths-hysteria-and-facts.pdf.

[8] UNDP. 2009. Human Development Report 2009-Overcoming Barriers: Human Mobility and Development. Retrieved October 8, 2020 from http://hdr.undp.org/sites/default/files/reports/269/hdr_2009_en_complete.pdf.

[9] A. Otoiu, E. Titan, and R. Dumitrescu. 2014. Internal and international migration: Is a dichotomous approach justified? Procedia Social and Behavioral Sciences 109 (2014), 1011-1015. DOI : https://doi.org/10.1016/j.sbspro.2013.12.581

[10] M. Bell and S. Muhidin. 2009. Cross-National Comparisons of Internal Migration. Retrieved October 8, 2020 from http:/hdr.undp.org/ sites/default/files/hdrp_2009_30.pdf.

[11] Global Migration Data Analysis Centre. 2020. Types of Migration. Retrieved October 8, 2020 from https://migrationdataportal.org/?i= stock_abs_\&t=2019.

[12] R. Skeldon. 2018. MRS No. 53-International Migration, Internal Migration, Mobility and Urbanization: Towards More Integrated Approaches. IOM Migration Research Series. United Nations, New York, NY.

[13] M. Van Ostaijen. 2016. Between migration and mobility discourses: The performative potential within "intra-European movement." Critical Policy Studies 11 (2016), 166-190. DOI : https://doi.org/10.1080/19460171.2015.1102751

[14] European Commission. 2019. Urban Agenda for the EU: Multi-Level Governance in Action. Retrieved October 8, 2020 from https: //ec.europa.eu/futurium/en/urban-agenda.

[15] C. Özden, C. R. Parsons, M. Schiff, and T. L. Walmsley. 2011. Where on Earth Is Everybody? Policy Research Working Paper No. 5709. World Bank, DC.

[16] D. Pitoski, T. J. Lampoltshammer, and P. Parycek. 2021. Human migration as a complex network: Appropriate abstraction, and the feasibility of Network Science tools. In Data Science-Analytics and Applications, P. Haber, T. Lampoltshammer, M. Mayr, and K. Plankensteiner (Eds.). Springer, 113-120. DOI: https://doi.org/10.1007/978-3-658-32182-6_17

[17] European Commission. 2018. Building a European Data Economy Policy. Retrieved October 8, 2020 from https://ec.europa.eu/digitalsingle-market/en/policies/75979/3489.

[18] Statistics Austria. 2020. Statistics Austria Open Data Catalogue. Retrieved October 8, 2020 from https://data.statistik.gv.at/web/meta. jsp?dataset=OGDEXT_BINNENWAND_1.

[19] R. Molontay and M. Nagy. 2019. Two decades of network science-As seen through the co-authorship network of network scientists. arXiv:1908.08478 [cs.SI]. DOI : https://doi.org/10.1145/3341161.3343685

[20] A. Barabasi. 1999. Emergence of scaling in random networks. Science 286, 5439 (1999), 509-512. DOI : https://doi.org/10.1126/science.286. 5439.509

[21] D. J. Watts and S. H. Strogatz. 1998. Collective dynamics of "small-world" networks. Nature 393, 6684 (1998), 440-442. DOI : https://doi. org $/ 10.1038 / 30918$

[22] M. Newman, A. Barabasi, and D Watts. 2006. The Structure and Dynamics of Networks. Princeton University Press, NJ.

[23] G. Fagiolo and M. Mastrorillo. 2013. International migration network: Topology and modeling. Physical Review E 88, 1 (2013), 012812 DOI : https://doi.org/10.1103/physreve.88.012812

[24] K. F. Davis, P. D’Odorico, F. Laio, and L. Ridolfi. 2013. Global spatio-temporal patterns in human migration: A complex network perspective. PLoS One 8 (2013), e53723. DOI : https://doi.org/10.1371/journal.pone.0053723

[25] E. Tranos, M. Gheasi, and P. Nijkamp. 2015. International migration: A global complex network. Environment and Planning B: Urban Analytics and City Science 42 (2015), 4-22. DOI : https://doi.org/10.1068/b39042

[26] The World Bank. 2017. Migration and Remittances Data. Retrieved October 8, 2020 from https://www.worldbank.org/en/topic/ migrationremittancesdiasporaissues/brief/migration-remittances-data.

[27] M. Peres, H. Xu, and G. Wu. 2016. Community evolution in international migration top1 networks. PLoS One 11 (2016), e0148615. DOI : https://doi.org/10.1371/journal.pone.0148615

[28] I. Porat and L. Penguigui. World migration degree. In Proceedings of the ERSA Conference. Article ersa14p60.

[29] V. Danchev and M. A. Porter. 2018. Neither global nor local: Heterogeneous connectivity in spatial network structures of world migration. Social Networks 53 (2018), 4-19. DOI : https://doi.org/10.1016/j.socnet.2017.06.003

[30] F. Aleskerov, N. Meshcheryakova, A. Rezyapova, and S. Shvydun. 2017. Network analysis of international migration. arXiv:1806.06705 [physics.soc-ph].

[31] R. Cerqueti, G. P. Clemente, and R. Grassi. 2018. A network-based measure of the socio-economic roots of the migration flows. Social Indicators Research 146 (2018), 187-204. DOI : https://doi.org/10.1007/s11205-018-1883-6

Digital Government: Research and Practice, Vol. 2, No. 3, Article 25. Publication date: July 2021. 
[32] M. Windzio. 2018. The network of global migration 1990-2013. Social Networks 53 (2018), 20-29. DOI : https://doi.org/10.1016/j.socnet. 2017.08.006

[33] V. Balàž and K. Karasovà. 2017. Geographical patterns in the intra-european migration before and after eastern enlargement: The connectivity approach. Fournal of Economics 65 (2017), 3-30.

[34] Z. Xu. 2017. The structure and dynamics of population migration among economic areas in the United States from 1990 to 2011. Papers in Regional Science 97 (2017), 785-800. DOI : https://doi.org/10.1111/pirs.12282

[35] X. Liu, R. Hollister, and C. Andris. 2018. Wealthy hubs and poor chains: Constellations in the U.S. urban migration system. In Agent-Based Models and Complexity Science in the Age of Geospatial Big Data. Advances in Geographic Information Science Book Series. Springer, $73-86$.

[36] T. Goldade, B. Charyyev, and M. H. Gunes. 2017. Network analysis of migration patterns in the United States. Complex Networks and Their Applications 6 (2017), 770-783. DOI : https://doi.org/10.1007/978-3-319-72150-7_62

[37] B. Charyyev and M. H. Gunes. 2019. Complex network of United States migration. Computational Social Networks 6 (2019), 1. DOI : https: //doi.org/10.1186/s40649-019-0061-6

[38] F. Li, Z. Feng, P. Li, and Z. You. 2017. Measuring directional urban spatial interaction in China: A migration perspective. PLoS One 12 (2017), e0171107. DOI : https://doi.org/10.1371/journal.pone.0171107

[39] Y. Sun and K. Pan. 2014. Prediction of the intercity migration of Chinese graduates. 2014. fournal of Statistical Mechanics: Theory and Experiment 2014 (2014), P12022. DOI : https://doi.org/10.1088/1742-5468/2014/12/p12022

[40] G. Maier and M. Vyborny. 2005. Internal Migration Between US States-A Social Network Analysis. Working Paper. University of Vienna.

[41] R. A. Manduca. 2014. Domestic Migration Networks in the United States. Massachusetts Institute of Technology, Cambridge, MA.

[42] V. L. Sciabolazza. 2018. Moving people: Network analysis of international migration flows. In Networks of International Trade and Investment, A. Amighini, S. Gorgoni, and M. Smith (Eds.). Vernon Press, Wilmington, DE, 249-296.

[43] D. Askar and T. House. 2010. Complex Patterns of Multiscale Human Mobility in United Kingdom. Working Paper. University of Warwick.

[44] C. Caudillo-Cos and R. Tapia-McClung. 2014. Patterns of internal migration of Mexican highly qualified population through network analysis. In Computational Science and Its Applications-ICCSA 2014. Lecture Notes in Computer Science, Vol. 8582. Springer, 169-184. DOI : https://doi.org/10.1007/978-3-319-09147-1_13

[45] M. Szell, R. Sinatra, G. Petri, S. Thurner, and V. Latora. 2012. Understanding mobility in a social petri dish. Scientific Reports 2 (2012), 457. DOI : https://doi.org/10.1038/srep00457

[46] D. Guo, X. Zhu, H. Jin, P. Gao, and C. Andris. 2012. Discovering spatial patterns in origin destination mobility data. Transactions in GIS 16, 3 (2012), 411-429. DOI : https://doi.org/10.1111/j.1467-9671.2012.01344.x

[47] B. Hawelka, I. Sitko, E. Beinat, S. Sobolevsky, P. Kazakopoulos, and C. Ratti. 2013. Geo-located Twitter as proxy for global mobility patterns. Cartography and Geographic Information Science 41, 3 (2013), 260-271. DOI : https://doi.org/10.1080/15230406.2014.890072

[48] F. B. Piel, A. J. Tatem, Z. Huang, S. Gupta, T. N. Williams, and D. J. Weatherall. 2014. Global migration and the changing distribution of sickle haemoglobin: A quantitative study of temporal trends between 1960 and 2000. Lancet Global Health 2, 2 (2014), e80-e89. DOI : https://doi.org/10.1016/S2214-109X(13)70150-5

[49] Y. Gong and M. Small. 2018. Epidemic spreading on metapopulation networks including migration and demographics. Chaos: An Interdisciplinary fournal of Nonlinear Science 28, 8 (2018), 083102. DOI : https://doi.org/10.1063/1.5021167

[50] G. Fagiolo and G. Santoni. 2014. Human-mobility networks, country income, and labor productivity. Network Science 3, 3 (2014), $377-407$. DOI : https://doi.org/10.2139/ssrn.2416574

[51] A. Garas, A. Lapatinas, and K. Poulios. 2016. The relation between migration and FDI in the OECD from a complex network perspective. Advances in Complex Systems 19, 6-7 (2016), 1650009. DOI : https://doi.org/10.1142/S0219525916500090

[52] P. Zhao, X. Liu, W. Shi, T. Jia, W. Li, and M. Chen. 2020. An empirical study on the intra-urban goods movement patterns using logistics big data. International fournal of Geographical Information Science 34, 6 (2020), 1089-1116. DOI : https://doi.org/10.1080/13658816.2018. 1520236

[53] F. Lillo and J. A. Molina Garay. 2019. The global remittance network: An inflow and outflow analysis. The fournal of Mathematical Sociology 43, 2 (2019), 59-75. DOI: https://doi.org/10.1080/0022250X.2018.1496917

[54] S. Lozano and E. Gutierrez. 2018. A complex network analysis of global tourism flows. International fournal of Tourism Research 20, 1 (2018), 588-604. DOI : https://doi.org/10.1002/jtr.2208

[55] D. Provenzano. 2020. The migration-tourism nexus in the EU28. Tourism Economics 26, 8 (2020), 1374-1393. DOI: https://doi.org/10. $1177 / 1354816620909994$

[56] Bundesanstalt Statistik Österreich. 2014. Standard-Dokumentation Metainformationen (Definitionen, Erläuterungen, Methoden, Qualität) zur Wanderungsstatistik. Retrieved October 8, 2020 from http:/www.statistik.at/web_de/wcmsprod/groups/gd/documents/stddok/ 029352.pdf\#pagemode=bookmarks.

[57] The World Bank. 2020. World Bank Open Data. Retrieved May 16, 2021 from https://data.worldbank.org/indicator/SP.POP.TOTL.

[58] A. Barrat, M. Barthélemy, R. Pastor-Satorras, and A. Vespignani. 2004. The architecture of complex weighted networks. Proceedings of the National Academy of Sciences of the United States of America 101 (2004), 3747-3752 DOI : https://doi.org/10.1073/pnas.0400087101 
[59] E. G. Ravenstein. 1889. The laws of migration. Journal of the Royal Statistical Society 52 (1889), 241-305. D0I: https://doi.org/10.2307/ 2979181

[60] D. Pitoski, T. J. Lampoltshammer, and P. Parycek. 2020. Supplementary Material for the Manuscript "Network Analysis of Internal Migration in Austria." figshare. Available at https://figshare.com. DOI : https://doi.org/10.6084/m9.figshare.12387122

[61] A. Clauset, C. R. Shalizi, and M. E. J. Newman. 2009. Power-law distributions in empirical data. SIAM Review 51, 4 (2009), $661-703$. DOI : https://doi.org/10.1137/070710111

[62] S. Brin and L. Page. 1998. The anatomy of a large-scale hypertextual Web search engine. Computer Networks and ISDN Systems 30 (1998), 107-117. DOI : https://doi.org/10.1016/S0169-7552(98)00110-X

[63] J. M. Kleinberg. 1999. Authoritative sources in a hyperlinked environment. Journal of the ACM 46 (1999), 604-632. DOI : https://doi.org/ $10.1145 / 324133.324140$

[64] T. Deguchi, K. Takahashi, H. Takayasu, and M. Takayasu. 2014. Hubs and authorities in the world trade network using a weighted HITS algorithm. PLoS One 9 (2014), e100338. DOI: https://doi.org/10.1371/journal.pone.0100338

[65] J. J. Bartholdi, P. Jarumaneeroj, and A. Ramudhin. 2016. A new connectivity index for container ports. Maritime Economics \& Logistics 18 (2016), 231-249. DOI : https://doi.org/10.1057/mel.2016.5

[66] T. Squartini, F. Picciolo, F. Ruzzenenti, D. Garlaschelli. 2013. Reciprocity of weighted networks. Scientific Reports 3 (2013), Article 2729. DOI : https://doi.org/10.1038/srep02729

[67] M. E. J. Newman. 2003. Mixing patterns in networks. Physical Review E 67, 2 (2003). DOI : https://doi.org/10.1103/PhysRevE.67.026126

[68] G. Csárdi and T. Nepusz. 2006. The iGraph Software Package for Complex Network Research. Retrieved October 8, 2020 from https: //cran.r-project.org/web/packages/igraph/igraph.pdf.

[69] M. E. J. Newman and M. Girvan. 2004. Finding and evaluating community structure in networks. Physical Review E 69 (2004), 026113. DOI : https://doi.org/10.1103/physreve.69.026113

[70] M. Rosvall, D. Axelsson, and C. T. Bergstrom. 2009. The map equation. European Physical fournal Special Topics 2009, 178 (2009), 13-23. DOI : https://doi.org/10.1140/epjst/e2010-01179-1

[71] V. D. Blondel, J.-L. Guillaume, R. Lambiotte, and E. Lefebvre. 2008. Fast unfolding of communities in large networks. fournal of Statistical Mechanics: Theory and Experiment 2008 (2008), P10008. DOI : https://doi.org/10.1088/1742-5468/2008/10/p10008

[72] Z. Yang, R. Algesheimer, and C. J. Tessone. 2016. A comparative analysis of community detection algorithms on artificial networks. Scientific Reports 6 (2016), 30750. DOI : https://doi.org/10.1038/srep30750

[73] A. Clauset, M. E. J. Newman, and C. Moore. 2004. Finding community structure in very large networks. Physical Review E 70 (2004), 066111. DOI : https://doi.org/10.1103/physreve.70.066111

[74] G. K. Zipf. 1946. The $\mathrm{P}_{1} \mathrm{P}_{2} / \mathrm{D}$ hypothesis: On the intercity movement of persons. American Sociological Review 11 (1946), 677-686, DOI : https://doi.org/10.2307/2087063

[75] P. Kaluza, A. Kolzsch, M. T. Gastner, and B. Blasius. 2010. The complex network of global cargo ship movements. fournal of the Royal Society Interface 7 (2010), 1093-1103. DOI : https://doi.org/10.1098/rsif.2009.0495

[76] F. Simini, M. C. González, A. Maritan, and A.-L. Barabási. 2012. A universal model for mobility and migration patterns. Nature 484 (2012), 96-100. DOI: https://doi.org/10.1038/nature10856

Received May 2020; revised October 2020; accepted January 2021 